\title{
A non-expanding transport distance for some structured equations
}

\author{
Nicolas Fournier* Benoît Perthame ${ }^{\dagger \ddagger}$
}

February 9, 2021

\begin{abstract}
Structured equations are a standard modeling tool in mathematical biology. They are integrodifferential equations where the unknown depends on one or several variables, representing the state or phenotype of individuals. A large literature has been devoted to many aspects of these equations and in particular to the study of measure solutions. Here we introduce a transport distance closely related to the Monge-Kantorovich distance, which appears to be non-expanding for several (mainly linear) examples of structured equations.
\end{abstract}

2010 Mathematics Subject Classification: 35A05; 35K55; 60J99; 28A33

Keywords and phrases: Transport distances; Monge-Kantorovich distance; Coupling; Structured equations; Mathematical biology.

\section{Introduction}

The subject of structured equations arises in several areas of biology and extends ordinary differential equations by including parameters chosen because they bring some influence on the population dynamics, see [11, 24, 30]. This leads to various integro-differential equations and partial differential equation (P.D.E.) which also appear in many other areas as physics, communication science and industry. Besides the interesting modeling issues, the questions which have been considered are about existence of solutions, entropy properties and, mostly, long term convergence to steady states, with possibly exponential rate of convergence. Another question concerns measure solutions, possibly after renormalization $[2,12]$. Furthermore, in the context of a nonlinear neuroscience problem, convergence of a particle system has recently been proved using transport costs with specific costs precisely adapted to the coefficients [19].

The present papers aims at showing that a simple variant of the Monge-Kantorovich transport distance appears to be non-expanding along several structured equations. These include the renewal equation and a few other models listed below.

We work in a state space that we denote by $\mathcal{J}$, which can be $[0, \infty),[0, \infty) \times T$ with $T$ a discrete torus, $[0, \infty)^{2}$ or $[0, \infty) \times \mathbb{R}^{d}$ and we always use a cost function $\varrho: \mathcal{J} \times \mathcal{J} \mapsto[0, \infty)$ which satisfies

\footnotetext{
${ }^{*}$ Sorbonne Université, CNRS, Laboratoire de Probabilité, Statistique et Modélisation, F-75005 Paris, France. Email: nicolas.fournier@sorbonne-universite.fr

†Sorbonne Université, CNRS, Université de Paris, Inria, Laboratoire Jacques-Louis Lions, F-75005 Paris, France. Email: benoit.perthame@sorbonne-universite.fr.

${ }^{\ddagger}$ B.P. has received funding from the European Research Council (ERC) under the European Union’s Horizon 2020 research and innovation programme (grant agreement No 740623).
} 
$\varrho(x, x)=0$ and $\varrho(x, y)=\varrho(y, x)>0$ for $x \neq y$ and will typically take the form $\varrho(y, x)=\min (|x-y|, a)$ for some $a>0$ chosen according to the equation at hand. We recall that for two probability measures $u_{1}, u_{2} \in \mathcal{P}(\mathcal{J})$, the transport cost is defined as

$$
\left\{\begin{array}{l}
\mathcal{T}_{\varrho}\left(u_{1}, u_{2}\right)=\inf _{v \in \mathcal{H}\left(u_{1}, u_{2}\right)} \iint \varrho(x, y) v(\mathrm{~d} x, \mathrm{~d} y), \\
\mathcal{H}\left(u_{1}, u_{2}\right)=\left\{v \in \mathcal{P}(\mathcal{J} \times \mathcal{J}) \quad \text { with marginals } u_{1} \text { and } u_{2}\right\} .
\end{array}\right.
$$

When $\varrho$ is a distance on $\mathcal{J}, \mathcal{T}_{\varrho}$ is a distance on $\mathcal{P}(\mathcal{J})$ and, with a slight abuse of language, one refers to the Monge-Kantorovich distance. Recent accounts about the theory can be found in the books $[32,1,31]$.

Our approach relies on the coupling method, see the survey paper [20]. The first example of use of the coupling method, to our knowledge, can be traced back to Dobrushin [14], where the nonlinear Vlasov equation is derived as mean-field limit of a deterministic system of interacting particles, making use of some transport cost. No P.D.E. is written for the coupling in [14], because everything may be expressed in terms of characteristics. See [21, Section 3] for a P.D.E. analogue to Dobrushin's argument. In the same spirit, the Euler equation is derived from a deterministic system of interacting vortices in Marchioro-Pulvirenti [23, Section 5.3], using also a coupling argument, see also [22] for a result with the strong transport distance $d_{\infty}$.

The paper is organized as follows. We begin with the renewal equation in order to present in details the results and method. Building on this, we extend the method to a system of renewal equations, some space-age structured equation, the multi-time renewal equation, the growth-fragmentation equation, and to an age-size coupled model. All these equations are linear. We complete our study with a model with sexual reproduction, which is quadratic, and generates new difficulties. Our setting is very general and does not use uniqueness of solutions, therefore we complete them with a technical appendix devoted to a uniqueness result by the Hilbert duality method when further regularity on the coefficients is assumed.

\section{The renewal equation}

Our first example, also the simpler, is the general renewal equation. It allows to introduce the method and to explain the choice of cost within the setting of the equation

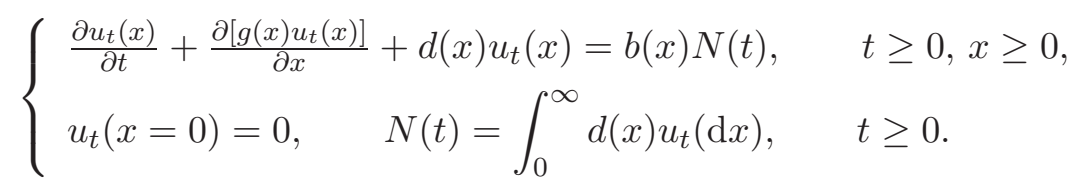

When $b=\delta_{0}$, the Dirac mass at 0 , and $g \equiv 1$, we find the classical renewal equation [17]. The more general version at hand is motivated by various models proposed in mathematical neuroscience, $[19,26,29]$. We assume that

$$
g, d \in C([0, \infty)), \quad b \in \mathcal{P}([0, \infty)), \quad \mathrm{g} \text { is non-increasing, } \quad g(0) \geq 0, \quad d \geq 0 .
$$

We will further suppose that

$$
\exists a>0 \quad \text { such that } a \leq \inf _{|x-y| \leq a} \frac{|x-y| \max (d(x), d(y))}{|d(x)-d(y)|} .
$$


Observe that this last condition holds true with $a=\min \left(a_{0}, 1\right)$ as soon as $a_{0}>0$, where

$$
a_{0}=\inf _{|x-y| \leq 1} \frac{|x-y| \max (d(x), d(y))}{|d(x)-d(y)|} .
$$

For example, $d(x)=\alpha+\beta x^{p}$ satisfies such a condition, provided $\alpha>0, \beta \geq 0$ and $p \geq 1$, as well as any Lipschitz and uniformly positive function.

Theorem 1 Assume (3)-(4). We consider the cost function on $(0, \infty) \times(0, \infty)$ defined by

$$
\varrho(x, y)=\min (a,|x-y|) .
$$

For any $u_{0}^{1}, u_{0}^{2} \in \mathcal{P}([0, \infty))$, there exists a pair of weak measure solutions $\left(u_{t}^{1}\right)_{t \geq 0},\left(u_{t}^{2}\right)_{t \geq 0} \subset \mathcal{P}([0, \infty))$ to (2) starting from $u_{0}^{1}$ and $u_{0}^{2}$, i.e., such that for $i=1,2$ and all $t \geq 0$,

$$
\int_{0}^{t} \int_{0}^{\infty} d(x) u_{s}^{i}(\mathrm{~d} x) \mathrm{d} s<\infty
$$

and for all $t \geq 0$, all $\varphi \in C_{c}^{1}([0, \infty))$,

$$
\int_{0}^{\infty} \varphi(x) u_{t}^{i}(\mathrm{~d} x)=\int_{0}^{\infty} \varphi(x) u_{0}^{i}(\mathrm{~d} x)+\int_{0}^{t} \int_{0}^{\infty}\left[g(x) \varphi^{\prime}(x)+d(x) \int_{0}^{\infty}(\varphi(z)-\varphi(x)) b(\mathrm{~d} z)\right] u_{s}^{i}(\mathrm{~d} x) \mathrm{d} s .
$$

Moreover, for all $t \geq 0$, we have

$$
\mathcal{T}_{\varrho}\left(u_{t}^{1}, u_{t}^{2}\right) \leq \mathcal{T}_{\varrho}\left(u_{0}^{1}, u_{0}^{2}\right)
$$

Notice that, even if we did not mention it, it follows from (6) that $u \in C_{\mathrm{w}}([0, \infty) ; \mathcal{P}([0, \infty))$. Also, the generality of this statement relies on the price that the solutions $u_{t}^{1}, u_{t}^{2}$ may depend on the choice of the pair $\left(u_{0}^{1}, u_{0}^{2}\right)$. However, when $g \in C_{b}^{1}$, the solutions are unique in distributional sense as proved in the appendix, and then the result is more standard. The regularity of $g$ can certainly be lowered in view of the theory developed in $[5,6,13]$.

Proof. We assume (3), fix $u_{0}^{1}, u_{0}^{2} \in \mathcal{P}([0, \infty))$ and consider any $v_{0} \in \mathcal{H}\left(u_{0}^{1}, u_{0}^{2}\right)$. There exists a family $\left(v_{t}\right)_{t \geq 0}$ of probability measures on $[0, \infty)^{2}$, starting from $v_{0}$, such that for all $t \geq 0$,

$$
\int_{0}^{t} \iint[d(x)+d(y)] v_{s}(\mathrm{~d} x, \mathrm{~d} y) \mathrm{d} s<\infty
$$

and which weakly solves

$$
\begin{aligned}
\frac{\partial v_{t}}{\partial t} & +\frac{\partial\left[g(x) v_{t}\right]}{\partial x}+\frac{\partial\left[g(y) v_{t}\right]}{\partial y}+\max (d(x), d(y)) v_{t}=b(x) \delta(x-y) \iint \min \left(d\left(x^{\prime}\right), d\left(y^{\prime}\right)\right) v_{t}\left(\mathrm{~d} x^{\prime}, \mathrm{d} y^{\prime}\right) \\
& +b(x) \int\left(d\left(x^{\prime}\right)-d(y)\right)_{+} v_{t}\left(\mathrm{~d} x^{\prime}, y\right)+b(y) \int\left(d\left(y^{\prime}\right)-d(x)\right)_{+} v_{t}\left(x, \mathrm{~d} y^{\prime}\right) .
\end{aligned}
$$


This means that for all $t \geq 0$, all $\varphi \in C_{c}^{1}\left([0, \infty)^{2}\right)$,

$$
\begin{aligned}
\iint \varphi(x, y) v_{t}(\mathrm{~d} x, \mathrm{~d} y)= & \iint \varphi(x, y) v_{0}(\mathrm{~d} x, \mathrm{~d} y)+\int_{0}^{t} \iint\left[g(x) \frac{\partial \varphi(x, y)}{\partial x}+g(y) \frac{\partial \varphi(x, y)}{\partial y}\right] v_{s}(\mathrm{~d} x, \mathrm{~d} y) \mathrm{d} s \\
& +\int_{0}^{t} \iiint[\varphi(z, z)-\varphi(x, y)] \min (d(x), d(y)) b(\mathrm{~d} z) v_{s}(\mathrm{~d} x, \mathrm{~d} y) \mathrm{d} s \\
& +\int_{0}^{t} \iiint[\varphi(z, y)-\varphi(x, y)](d(x)-d(y))_{+} b(\mathrm{~d} z) v_{s}(\mathrm{~d} x, \mathrm{~d} y) \mathrm{d} s \\
& +\int_{0}^{t} \iiint[\varphi(x, z)-\varphi(x, y)](d(y)-d(x))_{+} b(\mathrm{~d} z) v_{s}(\mathrm{~d} x, \mathrm{~d} y) \mathrm{d} s
\end{aligned}
$$

Using (7) and then (8) with a function $\varphi$ depending only on $x$, observing that $v_{0} \in \mathcal{H}\left(u_{0}^{1}, u_{0}^{2}\right)$ and that

$$
\min (d(x), d(y))+(d(x)-d(y))_{+}=d(x),
$$

we deduce that the first marginal $u_{t}^{1}(\mathrm{~d} x)=\int_{y \in[0, \infty)} v_{t}(\mathrm{~d} x, \mathrm{~d} y)$ satisfies (5)-(6). Similarly, the second marginal $u_{t}^{2}(\mathrm{~d} y)=\int_{x \in[0, \infty)} v_{t}(\mathrm{~d} x, \mathrm{~d} y)$ satisfies (5)-(6). And it holds that $v_{t} \in \mathcal{H}\left(u_{t}^{1}, u_{t}^{2}\right)$ for all $t \geq 0$.

The existence for (7)-(8) follows from classical arguments, using e.g., an approximate problem where $g, d$ are replaced by smooth and bounded functions, and from the following a priori tightness estimate. By the de la Vallée Poussin theorem, there exists a function $h:[0, \infty) \rightarrow[0, \infty)$ such that $\lim _{x \rightarrow \infty} h(x)=\infty$ and such that

$$
C:=\iint[h(x)+h(y)] v_{0}(\mathrm{~d} x, \mathrm{~d} y)+\int h(z) b(\mathrm{~d} z)<\infty .
$$

One can moreover choose $h$ smooth and satisfying $0 \leq h^{\prime} \leq 1$. Applying (8) with $\varphi(x, y)=h(x)+h(y)$, one immediately concludes that for all $t \geq 0$,

$$
\begin{aligned}
& \iint[h(x)+h(y)] v_{t}(\mathrm{~d} x, \mathrm{~d} y)=\iint[h(x)+h(y)] v_{0}(\mathrm{~d} x, \mathrm{~d} y)+\int_{0}^{t} \iint\left[g(x) h^{\prime}(x)+g(y) h^{\prime}(y)\right] v_{s}(\mathrm{~d} x, \mathrm{~d} y) \mathrm{d} s \\
&+\int_{0}^{t} \iint\left[d(x)\left(\int h(z) b(\mathrm{~d} z)-h(x)\right)+d(y)\left(\int h(z) b(\mathrm{~d} z)-h(y)\right)\right] v_{s}(\mathrm{~d} x, \mathrm{~d} y) \mathrm{d} s \\
& \leq C+2 \bar{C} t+\int_{0}^{t} \iint[d(x)(C-h(x))+d(y)(C-h(y))] v_{s}(\mathrm{~d} x, \mathrm{~d} y) \mathrm{d} s
\end{aligned}
$$

where $\bar{C}=\sup _{x \geq 0} g(x) h^{\prime}(x)$ is finite because $g$ is continuous and non-increasing and because $h^{\prime}$ is $[0,1]$-valued. Since now $d$ is continuous, non-negative and since $h$ increases to infinity, there is $L>0$ such that $d(x)(C-h(x)) \leq L-d(x) h(x) / 2$, whence finally

$$
\iint[h(x)+h(y)] v_{t}(\mathrm{~d} x, \mathrm{~d} y)+\frac{1}{2} \int_{0}^{t} \iint[d(x) h(x)+d(y) h(y)] v_{s}(\mathrm{~d} x, \mathrm{~d} y) \mathrm{d} s \leq C+2 \bar{C} t+2 L t .
$$

Since $\lim _{x \rightarrow \infty} h(x)=\infty$, this last a priori tightness estimate is sufficient to prove existence for (7)-(8).

We next fix $a>0$, set $\rho(x, y)=\min (|x-y|, a)$ and we choose a coupling $v_{0} \in \mathcal{H}\left(u_{0}^{1}, u_{0}^{2}\right)$ such that $\iint \rho(x, y) v_{0}(\mathrm{~d} x, \mathrm{~d} y)=\mathcal{T}_{\rho}\left(u_{0}^{1}, u_{0}^{2}\right)$. We apply (8) with $\varphi=\rho$ (or more precisely, firstly to some smooth and compactly supported approximation $\rho_{\varepsilon}$ of $\rho$ and then let $\left.\varepsilon \rightarrow 0\right)$. We notice that

$$
g(x) \frac{\partial \rho(x, y)}{\partial x}+g(y) \frac{\partial \rho(x, y)}{\partial y}=\mathbb{1}_{\{|x-y| \leq a\}} \operatorname{sgn}(x-y)[g(x)-g(y)] \leq 0
$$


because $g$ is non-increasing. Since $b$ is a probability measure, we obtain

$$
\begin{aligned}
\iint \rho(x, y) v_{t}(\mathrm{~d} x, \mathrm{~d} y) \leq & \mathcal{T}_{\rho}\left(u_{0}^{1}, u_{0}^{2}\right)-\int_{0}^{t} \iint \rho(x, y) \max (d(x), d(y)) v_{s}(\mathrm{~d} x, \mathrm{~d} y) \mathrm{d} s \\
& +\int_{0}^{t} \iiint\left[\rho(z, y)(d(x)-d(y))_{+}+\rho(x, z)(d(y)-d(x))_{+}\right] b(\mathrm{~d} z) v_{s}(\mathrm{~d} x, \mathrm{~d} y) \mathrm{d} s .
\end{aligned}
$$

Recalling that $\mathcal{T}_{\rho}\left(u_{t}^{1}, u_{t}^{2}\right) \leq \iint \rho(x, y) v_{t}(\mathrm{~d} x, \mathrm{~d} y)$, in order to complete the proof, it is therefore sufficient to verify the inequality, for all $x, y \geq 0$,

$$
I(x, y):=\frac{(d(x)-d(y))_{+}}{\max (d(x), d(y))} \int \varrho(z, y) b(\mathrm{~d} z)+\frac{(d(y)-d(x))_{+}}{\max (d(x), d(y))} \int \varrho(x, z) b(\mathrm{~d} z) \leq \varrho(x, y) .
$$

Since $\varrho(x, y)=\min (a,|x-y|)$ and since $b$ is a probability measure, we have

$$
I(x, y) \leq \frac{(d(x)-d(y))_{+}}{\max (d(x), d(y))} a+\frac{(d(y)-d(x))_{+}}{\max (d(x), d(y))} a=\frac{|d(x)-d(y)|}{\max (d(x), d(y))} a \leq \min (|x-y|, a) .
$$

Indeed, the last inequality is obvious if $|x-y| \geq a$ and follows from (4) otherwise.

\section{A system of renewal equations}

In mathematical biology, it is usual to describe the cell cycle, see [24, 30], using a system of renewal equations coupled at there boundaries as follows

$$
\begin{cases}\frac{\partial u_{t}(x, i)}{\partial t}+\frac{\partial\left[g_{i}(x) u_{t}(x, i)\right]}{\partial x}+d_{i}(x) u_{t}(x, i)=0, & t \geq 0, x \geq 0, i=1, \ldots, I, \\ u_{t}(x=0, i)=\int_{0}^{\infty} d_{i-1}(x) u_{t}(\mathrm{~d} x, i-1), & t \geq 0, i=1, \ldots, I,\end{cases}
$$

with the convention $u_{t}(x, 0)=u_{t}(x, I)$. In terms of stochastic processes, a cell with age $x$ in state $i$ ages chronologically (when, say, $g_{i} \equiv 1$ ) until, with rate $d_{i}(x)$, it changes state to $i+1$ where it starts with age $x=0$. So the state space is

$\mathcal{J}=[0, \infty) \times T$, with $T=\{1, \ldots, I\}$ the torus, i.e., states 0 and $I+1$ are identified to states $I$ and 1 .

We assume that for all $i \in T$,

$$
g_{i}, d_{i} \in C([0, \infty)), \quad g_{i} \text { is non-increasing, } \quad g_{i}(0) \geq 0, \quad d_{i} \geq 0,
$$

and that

$$
\exists a>0 \quad \text { such that } \quad a \leq \min _{1 \leq i \leq I} \inf _{|x-y| \leq a} \frac{|x-y| \max \left(d_{i}(x), d_{i}(y)\right)}{\left|d_{i}(x)-d_{i}(y)\right|} .
$$

This condition is satisfied if for all $i \in T$, there are $\alpha_{i}>0, \beta_{i} \geq 0$ and $p_{i} \geq 1$ such that $d_{i}(x)=$ $\alpha_{i}+\beta_{i} x^{p_{i}}$, or if all $d_{i}$ 's are Lipschitz and uniformly positive.

Theorem 2 Assume (11)-(12). We consider the cost on $\mathcal{J} \times \mathcal{J}$ defined by

$$
\varrho(x, i, y, j)=\min (a,|x-y|) \mathbb{I}_{\{i=j\}}+a \mathbb{I}_{\{i \neq j\}} .
$$


For any $u_{0}^{1}, u_{0}^{2} \in \mathcal{P}(\mathcal{J})$, there exists a pair of weak measure solutions $\left(u_{t}^{1}\right)_{t \geq 0},\left(u_{t}^{2}\right)_{t \geq 0} \subset \mathcal{P}(\mathcal{J})$ to $(10)$ starting from $u_{0}^{1}$ and $u_{0}^{2}$, i.e., such that for $j=1,2$ and all $t \geq 0$

$$
\int_{0}^{t} \int_{\mathcal{J}} d_{i}(x) u_{s}^{j}(\mathrm{~d} x, \mathrm{~d} i) \mathrm{d} s<\infty
$$

and for $j=1,2$, all $t \geq 0$ and all $\varphi \in C_{c}^{1}(\mathcal{J})$,

$$
\begin{aligned}
\int_{\mathcal{J}} \varphi(x, i) u_{t}^{j}(\mathrm{~d} x, \mathrm{~d} i)= & \int_{\mathcal{J}} \varphi(x, i) u_{0}^{j}(\mathrm{~d} x, \mathrm{~d} i) \\
& +\int_{0}^{t} \int_{\mathcal{J}}\left[g_{i}(x) \varphi^{\prime}(x, i)+d_{i}(x)[\varphi(0, i+1)-\varphi(x, i)]\right] u_{s}^{j}(\mathrm{~d} x, \mathrm{~d} i) \mathrm{d} s .
\end{aligned}
$$

Moreover, for all $t \geq 0$, we have

$$
\mathcal{T}_{\varrho}\left(u_{t}^{1}, u_{t}^{2}\right) \leq \mathcal{T}_{\varrho}\left(u_{0}^{1}, u_{0}^{2}\right)
$$

Note that if $u_{t}(x, i)$ is a strong solution to (10), then $u_{t}(\mathrm{~d} x, \mathrm{~d} i):=\sum_{k \in T} u_{t}(x, k) \mathrm{d} x \delta_{k}(\mathrm{~d} i)$ is a weak measure solution to (10). Note also that the comments after Theorem 1 apply here too.

Proof. We assume (11)-(12) and consider any coupling $v_{0} \in \mathcal{H}\left(u_{0}^{1}, u_{0}^{2}\right)$. There exists a family $\left(v_{t}\right)_{t \geq 0}$ of probability measures on $\mathcal{J}^{2}$ such that for all $t \geq 0$,

$$
\int_{0}^{t} \iint\left[d_{i}(x)+d_{j}(y)\right] v_{s}(\mathrm{~d} x, \mathrm{~d} i, \mathrm{~d} y, \mathrm{~d} j) \mathrm{d} s<\infty,
$$

and which weakly solves

$$
\begin{aligned}
\frac{\partial v_{t}}{\partial t}+ & \frac{\partial\left[g_{i}(x) v_{t}\right]}{\partial x}+\frac{\partial\left[g_{j}(y) v_{t}\right]}{\partial y}+\left[\left(d_{i}(x) \vee d_{j}(y)\right) \mathbb{I}_{\{i=j\}}+\left(d_{i}(x)+d_{j}(y)\right) \mathbb{I}_{\{i \neq j\}}\right] v_{t}= \\
& \delta_{(0,0)}(x, y) \mathbb{I}_{\{i=j\}} \iint\left(d_{i-1}\left(x^{\prime}\right) \wedge d_{i-1}\left(y^{\prime}\right)\right) v_{t}\left(\mathrm{~d} x^{\prime}, i-1, \mathrm{~d} y^{\prime}, j-1\right) \\
& +\delta_{0}(x) \mathbb{I}_{\{j=i-1\}} \int\left(d_{i-1}\left(x^{\prime}\right)-d_{j}(y)\right)_{+} v_{t}\left(\mathrm{~d} x^{\prime}, i-1, y, j\right) \\
& +\delta_{0}(y) \mathbb{I}_{\{i=j-1\}} \int\left(d_{j-1}\left(y^{\prime}\right)-d_{i}\left(x^{\prime}\right)\right)_{+} v_{t}\left(x, i, \mathrm{~d} y^{\prime}, j-1\right) \\
& +\delta_{0}(x) \mathbb{I}_{\{j \neq i-1\}} \int d_{i-1}\left(x^{\prime}\right) v_{t}\left(\mathrm{~d} x^{\prime}, i-1, y, j\right)+\delta_{0}(y) \mathbb{I}_{\{i \neq j-1\}} \int d_{j-1}\left(y^{\prime}\right) v_{t}\left(x, i, \mathrm{~d} y^{\prime}, j-1\right) .
\end{aligned}
$$

This means that for all $\varphi \in C_{c}^{1}\left(\mathcal{J}^{2}\right)$, all $t \geq 0$,

$$
\begin{aligned}
& \iint \varphi(x, i, y, j) v_{t}(\mathrm{~d} x, \mathrm{~d} i, \mathrm{~d} y, \mathrm{~d} j)=\iint \varphi(x, i, y, j) v_{0}(\mathrm{~d} x, \mathrm{~d} i, \mathrm{~d} y, \mathrm{~d} j) \\
&+\int_{0}^{t} \iint\left[g_{i}(x) \frac{\partial \varphi(x, i, y, j)}{\partial x}+g_{j}(y) \frac{\partial \varphi(x, i, y, j)}{\partial y}\right] v_{s}(\mathrm{~d} x, \mathrm{~d} i, \mathrm{~d} y, \mathrm{~d} j) \mathrm{d} s \\
&+\int_{0}^{t} \iint\left([\varphi(0, i+1,0, j+1)-\varphi(x, i, y, j)] \mathbb{I}_{\{i=j\}}\left(d_{i}(x) \wedge d_{j}(y)\right)\right. \\
&+[\varphi(0, i+1, y, j)-\varphi(x, i, y, j)] \mathbb{I}_{\{i=j\}}\left(d_{i}(x)-d_{j}(y)\right)_{+} \\
&+[\varphi(x, i, 0, j+1)-\varphi(x, i, y, j)] \mathbb{I}_{\{i=j\}}\left(d_{j}(y)-d_{i}(x)\right)_{+} \\
&+[\varphi(0, i+1, y, j)-\varphi(x, i, y, j)] \mathbb{I}_{\{i \neq j\}} d_{i}(x) \\
&\left.+[\varphi(x, i, 0, j+1)-\varphi(x, i, y, j)] \mathbb{I}_{\{i \neq j\}} d_{j}(y)\right) v_{s}(\mathrm{~d} x, \mathrm{~d} i, \mathrm{~d} y, \mathrm{~d} j) \mathrm{d} s .
\end{aligned}
$$


Using (15) and (16) with a function $\varphi$ depending only on $(x, i)$ (or $(y, j)$ ), we see that the first marginal $u_{t}^{1}(\mathrm{~d} x, \mathrm{~d} i)=\int_{(y, j) \in \mathcal{J}} v_{t}(\mathrm{~d} x, \mathrm{~d} i, \mathrm{~d} y, \mathrm{~d} j)$ (or the second marginal $\left.u_{t}^{2}(\mathrm{~d} y, \mathrm{~d} j)=\int_{(x, i) \in \mathcal{J}} v_{t}(\mathrm{~d} x, \mathrm{~d} i, \mathrm{~d} y, \mathrm{~d} j)\right)$ satisfies (13) and (14).

As before, the existence for (15)-(16) follows from classical arguments, using an approximate problem where $g_{i}, d_{i}$ are replaced by smooth and bounded functions, and from the following a priori tightness estimate. By the de la Vallée Poussin theorem, there exists a function $h:[0, \infty) \rightarrow[0, \infty)$ such that $\lim _{x \rightarrow \infty} h(x)=\infty$ and such that

$$
C:=\iint[h(x)+h(y)] v_{0}(\mathrm{~d} x, \mathrm{~d} i, \mathrm{~d} y, \mathrm{~d} j)<\infty .
$$

One can moreover choose $h$ smooth, satisfying $h(0)=0$ and $0 \leq h^{\prime} \leq 1$. Applying (16) with $\varphi(x, i, y, j)=h(x)+h(y)$, one immediately concludes that for all $t \geq 0$,

$$
\begin{aligned}
\iint[h(x)+h(y)] v_{t}(\mathrm{~d} x, \mathrm{~d} i, \mathrm{~d} y, \mathrm{~d} j)= & \iint[h(x)+h(y)] v_{0}(\mathrm{~d} x, \mathrm{~d} i, \mathrm{~d} y, \mathrm{~d} j) \\
& +\int_{0}^{t} \iint\left[g_{i}(x) h^{\prime}(x)+g_{j}(y) h^{\prime}(y)\right] v_{s}(\mathrm{~d} x, \mathrm{~d} i, \mathrm{~d} y, \mathrm{~d} j) \mathrm{d} s \\
& -\int_{0}^{t} \iint\left[d_{i}(x) h(x)+d_{j}(y) h(y)\right] v_{s}(\mathrm{~d} x, \mathrm{~d} i, \mathrm{~d} y, \mathrm{~d} j) \mathrm{d} s .
\end{aligned}
$$

Setting $\bar{C}=\sup _{i \in T, x \geq 0} g_{i}(x) h^{\prime}(x)$, which is finite because $g_{i}$ is continuous, non-increasing and because $h^{\prime}$ is $[0,1]$-valued, we end up with

$$
\iint[h(x)+h(y)] v_{t}(\mathrm{~d} x, \mathrm{~d} i, \mathrm{~d} y, \mathrm{~d} j)+\int_{0}^{t} \iint\left[d_{i}(x) h(x)+d_{j}(y) h(y)\right] v_{s}(\mathrm{~d} x, \mathrm{~d} i, \mathrm{~d} y, \mathrm{~d} j) \mathrm{d} s \leq C+2 \bar{C} t .
$$

This last a priori tightness estimate is sufficient to prove existence for (15)-(16).

We now apply (16) with $\varphi=\varrho$, after regularization as before, where we recall that $\varrho(x, i, y, j)=$ $\min (a,|x-y|) \mathbb{I}_{\{i=j\}}+a \mathbb{I}_{\{i \neq j\}}$. Since $g_{i}$ is non-increasing, we have

$$
g_{i}(x) \frac{\partial \varrho(x, i, y, j)}{\partial x}+g_{j}(y) \frac{\partial \varrho(x, i, y, j)}{\partial y}=\mathbb{I}_{\{i=j\}} \mathbb{I}_{\{|x-y| \leq a\}} \operatorname{sgn}(x-y)\left[g_{i}(x)-g_{j}(y)\right] \leq 0 .
$$

Hence we find, choosing $v_{0}$ such that $\iint \varrho(x, i, y, j) v_{0}(\mathrm{~d} x, \mathrm{~d} i, \mathrm{~d} y, \mathrm{~d} j)=\mathcal{T}_{\rho}\left(u_{0}^{1}, u_{0}^{2}\right)$,

$$
\begin{aligned}
\iint \varrho(x, i, y, j) v_{t}(\mathrm{~d} x, \mathrm{~d} i, \mathrm{~d} y, \mathrm{~d} j) \\
\leq \mathcal{T}_{\rho}\left(u_{0}^{1}, u_{0}^{2}\right)+\int_{0}^{t} \iint\left([\varrho(0, i+1,0, j+1)-\varrho(x, i, y, j)] \mathbb{I}_{\{i=j\}}\left(d_{i}(x) \wedge d_{j}(y)\right)\right. \\
+[\varrho(0, i+1, y, j)-\varrho(x, i, y, j)] \mathbb{I}_{\{i=j\}}\left(d_{i}(x)-d_{j}(y)\right)_{+} \\
+[\varrho(x, i, 0, j+1)-\varrho(x, i, y, j)] \mathbb{I}_{\{i=j\}}\left(d_{j}(y)-d_{i}(x)\right)_{+} \\
+[\varrho(0, i+1, y, j)-\varrho(x, i, y, j)] \mathbb{I}_{\{i \neq j\}} d_{i}(x) \\
\left.+[\varrho(x, i, 0, j+1)-\varrho(x, i, y, j)] \mathbb{I}_{\{i \neq j\}} d_{j}(y)\right) v_{s}(\mathrm{~d} x, \mathrm{~d} i, \mathrm{~d} y, \mathrm{~d} j) \mathrm{d} s .
\end{aligned}
$$


With our choice of $\varrho$, the two last lines are non-positive. We thus arrive at

$$
\iint \varrho(x, i, y, j) v_{t}(\mathrm{~d} x, \mathrm{~d} i, \mathrm{~d} y, \mathrm{~d} j) \leq \mathcal{T}_{\rho}\left(u_{0}^{1}, u_{0}^{2}\right)-\int_{0}^{t} \iint \mathbb{I}_{\{i=j\}} \Delta_{i}(x, y) v_{s}(\mathrm{~d} x, \mathrm{~d} i, \mathrm{~d} y, \mathrm{~d} j) \mathrm{d} s,
$$

where

$$
\begin{aligned}
\Delta_{i}(x, y) & =\left(d_{i}(x) \vee d_{i}(y)\right)(|x-y| \wedge a)-\left(d_{i}(x)-d_{i}(y)\right)_{+} a-\left(d_{i}(y)-d_{i}(x)\right)_{+} a \\
& =\left(d_{i}(x) \vee d_{i}(y)\right)(|x-y| \wedge a)-\left|d_{i}(x)-d_{i}(y)\right| a
\end{aligned}
$$

Next, we check that $\Delta_{i}$ is always non-negative. If $|x-y| \geq a$, this is obvious. If $|x-y| \leq a$, this follows from (12). Since $\mathcal{T}_{\rho}\left(u_{t}^{1}, u_{t}^{2}\right) \leq \iint \rho(x, i, y, j) v_{t}(\mathrm{~d} x, \mathrm{~d} i, \mathrm{~d} y, \mathrm{~d} j)$, the proof is complete.

\section{$3 \quad$ Space and age structure}

Next, we consider an example similar to that in the previous section, when the discrete parameter $i$ is replaced by a continuous parameter $z \in \mathbb{R}^{d}$, which represents space or a physiological trait. The formalism makes the link with the heat equation through a standard physical process used in particular to describe diffusion or anomalous diffusion, see recent analyses in $[28,8,3]$. We depart from the equation

$$
\left\{\begin{array}{l}
\varepsilon^{2} \frac{\partial u_{t}(x, z)}{\partial t}+\frac{\partial u_{t}(x, z)}{\partial x}+d(x) u_{t}(x, z)=0, \quad t \geq 0, x>0, z \in \mathbb{R}^{d} \\
u_{t}(x=0, z)=\int_{0}^{\infty} \int_{\mathbb{R}^{d}} d(x) u_{t}(\mathrm{~d} x, z+\varepsilon \eta) k(\mathrm{~d} \eta), \quad t \geq 0, z \in \mathbb{R}^{d}
\end{array}\right.
$$

This equation models particles characterized by their age $x$ and position $z$. When in state $x, z$, the particle's age $x$ grows linearly until there is a jump, at rate $d(x)$, resulting in the particle moving from $z$ to $z-\varepsilon \eta$ (with $\eta$ chosen according to the probability density $k$ ). At each jump, the age is reset to 0 . Hence the state space is here $\mathcal{J}=[0, \infty) \times \mathbb{R}^{d}$. Under a few assumptions, it is known that, for $u_{\varepsilon}$ the solution to $(17), U_{\varepsilon}(t, z)=\int_{0}^{\infty} u_{\varepsilon}(t, x, z) \mathrm{d} x$ converges, as $\varepsilon \rightarrow 0$, to the solution of the heat equation in $\mathbb{R}^{d}$.

We assume that

$$
d \in C([0, \infty)), \quad d \geq 0, \quad k \in \mathcal{P}\left(\mathbb{R}^{d}\right),
$$

and, again, that

$$
\exists a>0 \quad \text { such that } \quad a \leq \inf _{\mid x-y] \leq a} \frac{|x-y| \max (d(x), d(y))}{|d(x)-d(y)|} .
$$

Theorem 3 Assume (18)-(19) and fix $\varepsilon>0$. We consider the cost on $\mathcal{J} \times \mathcal{J}$ defined by

$$
\varrho(x, z, y, r)=\min (a,|x-y|+|z-r|) .
$$

For any $u_{0}^{1}, u_{0}^{2} \in \mathcal{P}(\mathcal{J})$, there exists a pair of weak measure solutions $\left(u_{t}^{1}\right)_{t \geq 0},\left(u_{t}^{2}\right)_{t \geq 0} \subset \mathcal{P}(\mathcal{J})$ to $(17)$ starting from $u_{0}^{1}$ and $u_{0}^{2}$, i.e., such that for $i=1,2$ and all $t \geq 0$,

$$
\int_{0}^{t} \int_{\mathcal{J}} d(x) u_{s}^{i}(\mathrm{~d} x, \mathrm{~d} z) \mathrm{d} s<\infty
$$


and for $i=1,2$, all $t \geq 0$ and all $\varphi \in C_{c}^{1}(\mathcal{J})$,

$$
\begin{aligned}
\int_{\mathcal{J}} \varphi(x, z) u_{t}^{i}(\mathrm{~d} x, \mathrm{~d} z)=\int_{\mathcal{J}} \varphi(x, z) u_{0}^{i}(\mathrm{~d} x, \mathrm{~d} z) \\
\quad+\varepsilon^{-2} \int_{0}^{t} \int_{\mathcal{J}}\left[\frac{\partial \varphi(x, z)}{\partial x}+d(x) \int_{\mathbb{R}^{d}}[\varphi(0, z-\varepsilon \eta)-\varphi(x, z)] k(\mathrm{~d} \eta)\right] u_{s}^{i}(\mathrm{~d} x, \mathrm{~d} z) \mathrm{d} s .
\end{aligned}
$$

Moreover, for all $t \geq 0$, we have

$$
\mathcal{T}_{\varrho}\left(u_{t}^{1}, u_{t}^{2}\right) \leq \mathcal{T}_{\varrho}\left(u_{0}^{1}, u_{0}^{2}\right)
$$

Proof. We assume (18)-(19), consider $u_{0}^{1}, u_{0}^{2} \in \mathcal{P}(\mathcal{J})$ and a coupling $v_{0} \in \mathcal{H}\left(u_{0}^{1}, u_{0}^{2}\right)$. There exists a family $\left(v_{t}\right)_{t \geq 0}$ of probability measures on $\mathcal{J}^{2}$ such that for all $t \geq 0$

$$
\int_{0}^{t} \iint[d(x)+d(y)] v_{t}(\mathrm{~d} x, \mathrm{~d} z, \mathrm{~d} y, \mathrm{~d} r)<\infty
$$

and which weakly solve

$$
\begin{aligned}
\varepsilon^{2} \frac{\partial v_{t}}{\partial t}+\frac{\partial v_{t}}{\partial x}+ & \frac{\partial v_{t}}{\partial y}+(b(x) \vee b(y)) v_{t}= \\
& \delta(x) \delta(y) \int_{0}^{\infty} \int_{0}^{\infty} \int_{\mathbb{R}^{d}}\left(b\left(x^{\prime}\right) \wedge b\left(y^{\prime}\right)\right) v_{t}\left(\mathrm{~d} x^{\prime}, z+\varepsilon \eta, \mathrm{d} y^{\prime}, r+\varepsilon \eta\right) k(\eta) \mathrm{d} \eta \\
& +\delta(x) \int_{\mathcal{J}}\left(b\left(x^{\prime}\right)-b(y)\right)_{+} v_{t}\left(\mathrm{~d} x^{\prime}, z+\varepsilon \eta, y, r+\varepsilon \eta\right) k(\eta) \mathrm{d} \eta \\
& +\delta(y) \int_{\mathcal{J}}\left(b\left(y^{\prime}\right)-b(x)\right)_{+} v_{t}\left(x, z+\varepsilon \eta, \mathrm{d} y^{\prime}, r+\varepsilon \eta\right) k(\eta) \mathrm{d} \eta
\end{aligned}
$$

This means that for all $\varphi \in C_{c}^{1}\left(\mathcal{J}^{2}\right)$, all $t \geq 0$,

$$
\begin{aligned}
\iint \varphi(x, z, y, r) v_{t}(\mathrm{~d} x, \mathrm{~d} z, \mathrm{~d} y, \mathrm{~d} r)=\iint \varphi(x, z, y, r) v_{0}(\mathrm{~d} x, \mathrm{~d} z, \mathrm{~d} y, \mathrm{~d} r) \\
+\varepsilon^{-2} \int_{0}^{t} \iint\left[\frac{\partial \varphi(x, z, y, r)}{\partial x}+\frac{\partial \varphi(x, z, y, r)}{\partial y}\right] v_{s}(\mathrm{~d} x, \mathrm{~d} z, \mathrm{~d} y, \mathrm{~d} r) \mathrm{d} s \\
+\varepsilon^{-2} \int_{0}^{t} \iint\left(\left[(d(x) \wedge d(y)) \int_{\mathbb{R}^{d}}[\varphi(0, z-\varepsilon \eta, 0, r-\varepsilon \eta)-\varphi(x, z, y, r)] k(\mathrm{~d} \eta)\right.\right. \\
+(d(x)-d(y))_{+} \int_{\mathbb{R}^{d}}[\varphi(0, z-\varepsilon \eta, y, r)-\varphi(x, z, y, r)] k(\mathrm{~d} \eta) \\
\left.+(d(y)-d(x))_{+} \int_{\mathbb{R}^{d}}[\varphi(x, z, 0, r-\varepsilon \eta)-\varphi(x, z, y, r)] k(\mathrm{~d} \eta)\right) v_{s}(\mathrm{~d} x, \mathrm{~d} z, \mathrm{~d} y, \mathrm{~d} r) \mathrm{d} s
\end{aligned}
$$

Using (22) and (23) with a function $\varphi$ depending only on $(x, z)$ (or $(y, r)$ ), we see that the first marginal $u_{t}^{1}(\mathrm{~d} x, \mathrm{~d} z)=\int_{(y, r) \in \mathcal{J}} v_{t}(\mathrm{~d} x, \mathrm{~d} z, \mathrm{~d} y, \mathrm{~d} r)$ (and the second one $\left.u_{t}^{2}(\mathrm{~d} y, \mathrm{~d} r)=\int_{(x, z) \in \mathcal{J}} v_{t}(\mathrm{~d} x, \mathrm{~d} z, \mathrm{~d} y, \mathrm{~d} r)\right)$ satisfies (20) and (21). 
The existence for (15)-(16) follows as usual from the following a priori tightness estimate. By the de la Vallée Poussin theorem, there is a function $h:[0, \infty) \rightarrow[0, \infty)$ such that $\lim _{x \rightarrow \infty} h(x)=\infty$ and

$$
C:=\iint[h(x)+h(y)+h(|z|)+h(|r|)] v_{0}(\mathrm{~d} x, \mathrm{~d} z, \mathrm{~d} y, \mathrm{~d} r)+\int h(\varepsilon|\eta|) k(\mathrm{~d} \eta)<\infty .
$$

One can moreover choose $h$ smooth, satisfying $h(0)=0$ and $0 \leq h^{\prime} \leq 1$. Applying (23) with $\varphi(x, z, y, r)=h(x)+h(y)+h(|z|)+h(|r|)$, one immediately concludes that for all $t \geq 0$,

$$
\begin{array}{r}
\iint[h(x)+h(y)+h(|z|)+h(|r|)] v_{t}(\mathrm{~d} x, \mathrm{~d} z, \mathrm{~d} y, \mathrm{~d} r) \leq C+\varepsilon^{-2} \int_{0}^{t} \iint\left[h^{\prime}(x)+h^{\prime}(y)\right] v_{s}(\mathrm{~d} x, \mathrm{~d} z, \mathrm{~d} y, \mathrm{~d} r) \mathrm{d} s \\
+\varepsilon^{-2} \int_{0}^{t} \iint\left[d(x) \int[h(0)+h(|z-\varepsilon \eta|)-h(x)-h(|z|)] k(\mathrm{~d} \eta)\right. \\
\left.+d(y) \int[h(0)+h(|r-\varepsilon \eta|)-h(y)-h(|r|)] k(\mathrm{~d} \eta)\right] v_{s}(\mathrm{~d} x, \mathrm{~d} z, \mathrm{~d} y, \mathrm{~d} r) \mathrm{d} s .
\end{array}
$$

Using that $h^{\prime}$ is $[0,1]$-valued and that $h(0)=0$, we observe that

$$
\int[h(0)+h(|z-\varepsilon \eta|)-h(x)-h(|z|)] k(\mathrm{~d} \eta) \leq \int h(|\varepsilon \eta|)(\mathrm{d} \eta)-h(x) \leq C-h(x) .
$$

Using now that $d(x)[C-h(x)] \leq L-d(x) h(x) / 2$ for some constant $L>0$, we end up with

$$
\begin{gathered}
\iint[h(x)+h(y)+h(|z|)+h(|r|)] v_{t}(\mathrm{~d} x, \mathrm{~d} z, \mathrm{~d} y, \mathrm{~d} r)+\frac{1}{2 \varepsilon^{2}} \int_{0}^{t} \iint[d(x) h(x)+d(y) h(y)] v_{s}(\mathrm{~d} x, \mathrm{~d} z, \mathrm{~d} y, \mathrm{~d} r) \mathrm{d} s \\
\leq C+2 \varepsilon^{-2} t+2 \varepsilon^{-2} L t .
\end{gathered}
$$

This a priori tightness estimate is sufficient, as usual, to prove existence for (22)-(23).

We now apply $(23)$ with $\varphi=\varrho$, where we recall that $\varrho(x, z, y, r)=\min (a,|x-y|+|z-r|)$. Since

$$
\frac{\partial \varrho(x, z, y, r)}{\partial x}+\frac{\partial \varrho(x, z, y, r)}{\partial y}=0
$$

we find, choosing $v_{0}$ such that $\iint \varrho(x, z, y, r) v_{0}(\mathrm{~d} x, \mathrm{~d} z, \mathrm{~d} y, \mathrm{~d} r)=\mathcal{T}_{\rho}\left(u_{0}^{1}, u_{0}^{2}\right)$,

$$
\iint \varrho(x, z, y, r) v_{t}(\mathrm{~d} x, \mathrm{~d} z, \mathrm{~d} y, \mathrm{~d} r) \leq \mathcal{T}_{\rho}\left(u_{0}^{1}, u_{0}^{2}\right)+\varepsilon^{-2} \int_{0}^{t} \iint \Delta(x, z, y, r) v_{s}(\mathrm{~d} x, \mathrm{~d} z, \mathrm{~d} y, \mathrm{~d} r) \mathrm{d} s,
$$

where

$$
\begin{aligned}
\Delta(x, z, y, r)= & (d(x) \wedge d(y)) \int_{\mathbb{R}^{d}}[\varrho(0, z-\varepsilon \eta, 0, r-\varepsilon \eta)-\varrho(x, z, y, r)] k(\mathrm{~d} \eta) \\
& +(d(x)-d(y))_{+} \int_{\mathbb{R}^{d}}[\varrho(0, z-\varepsilon \eta, y, r)-\varrho(x, z, y, r)] k(\mathrm{~d} \eta) \\
& +(d(y)-d(x))_{+} \int_{\mathbb{R}^{d}}[\varrho(x, z, 0, r-\varepsilon \eta)-\varrho(x, z, y, r)] k(\mathrm{~d} \eta) .
\end{aligned}
$$

Since $\mathcal{T}_{\rho}\left(u_{t}^{1}, u_{t}^{2}\right) \leq \iint \rho(x, z, y, r) v_{t}(\mathrm{~d} x, \mathrm{~d} z, \mathrm{~d} y, \mathrm{~d} r)$, it thus only remains to check that $\Delta$ is always non-positive. But we have, assuming e.g. that $d(x) \geq d(y)$,

$$
\begin{aligned}
\Delta(x, z, y, r)= & -d(x)[(|x-y|+|z-r|) \wedge a]+d(y)(|z-r| \wedge a) \\
& +(d(x)-d(y)) \int_{\mathbb{R}^{d}}[(|y|+|z-\varepsilon \eta-r|) \wedge a] k(\mathrm{~d} \eta) \\
\leq & -d(x)[(|x-y|+|z-r|) \wedge a]+d(y)(|z-r| \wedge a)+(d(x)-d(y)) a .
\end{aligned}
$$


If $|x-y|+|z-r| \geq a$, we have

$$
\Delta(x, z, y, r) \leq-d(x) a+d(y) a+(d(x)-d(y)) a=0 .
$$

If $|x-y|+|z-r| \leq a$, still assuming that $d(x) \geq d(y)$, we have

$$
\begin{aligned}
\Delta(x, z, y, r) & \leq-d(x)(|x-y|+|z-r|)+d(y)|z-r|+(d(x)-d(y)) a \\
& \leq-d(x)|x-y|+(d(x)-d(y)) a \leq 0
\end{aligned}
$$

thanks to (19), since $|x-y|+|z-r| \leq a$ implies that $|x-y| \leq a$. Therefore, we always have $\Delta(x, z, y, r) \leq 0$ and the proof is complete.

\section{The multiple time renewal equation}

Several applications use multi-time renewal equations to describe a population density subjected to aging or to time-evolution. Recently, for evaluating the efficiency of tracing softwares, it was used to take into account secondary infections, see [18]. In neuroscience, the interpretation is that neurones keep memory of their last spikes in the process of deciding when to fire again, see [10]. For two times memory, the equation reads

$$
\begin{cases}\frac{\partial u_{t}\left(x_{1}, x_{2}\right)}{\partial t}+\frac{\partial u_{t}\left(x_{1}, x_{2}\right)}{\partial x_{1}}+\frac{\partial u_{t}\left(x_{1}, x_{2}\right)}{\partial x_{2}}+d\left(x_{1}, x_{2}\right) u_{t}\left(x_{1}, x_{2}\right)=0, & x_{2} \geq x_{1} \geq 0, t \geq 0, \\ u_{t}\left(x_{1}=0, x_{2}\right)=\int_{x_{1}}^{\infty} d\left(x_{2}, z\right) u_{t}\left(x_{2}, \mathrm{~d} z\right), & x_{2} \geq 0, t \geq 0 .\end{cases}
$$

An example of stochastic interpretation is that particles are individuals producing events at a rate depending on the ages of its two last events. Here the variables $x_{1}$ and $x_{2}$ represent the ages of the two last events, so these ages increase linearly until, at rate $d\left(x_{1}, x_{2}\right)$, they are reset to the values $\left(0, x_{1}\right)$. Hence our state space is now $\mathcal{J}=\left\{\left(x_{1}, x_{2}\right) \in[0, \infty)^{2}: x_{2}>x_{1}\right\}$. We assume that

$$
d \in C(\mathcal{J}), \quad d \geq 0
$$

and that

$$
\exists a>0 \quad \text { such that } a \leq \inf _{2\left|x_{1}-\widetilde{x}_{1}\right|+\left|x_{2}-\widetilde{x}_{2}\right| \leq a} \frac{\left(\left|x_{1}-\widetilde{x}_{1}\right|+\left|x_{2}-\tilde{x}_{2}\right|\right) \max \left(d\left(x_{1}, x_{2}\right), d\left(\widetilde{x}_{1}, \widetilde{x}_{2}\right)\right)}{\left|d\left(x_{1}, x_{2}\right)-d\left(\widetilde{x}_{1}, \widetilde{x}_{2}\right)\right|} .
$$

One can check that $d\left(x_{1}, x_{2}\right)=\alpha+\beta x_{1}^{p_{1}}+\gamma x_{2}^{p_{2}}$, with $\alpha>0, \beta \geq 0, \gamma \geq 0, p_{1} \geq 1$ and $p_{2} \geq 1$ satisfies such an assumption, as well as Lipschitz and uniformly positive functions.

Theorem 4 We assume (25)-(26). We consider the cost on $\mathcal{J} \times \mathcal{J}$ defined by

$$
\varrho\left(x_{1}, x_{2}, \widetilde{x}_{1}, \widetilde{x}_{2}\right)=\left[2\left|x_{1}-\widetilde{x}_{1}\right|+\left|x_{2}-\widetilde{x}_{2}\right|\right] \wedge a .
$$

For any $u_{0}^{1}, u_{0}^{2} \in \mathcal{P}(\mathcal{J})$, there exists a pair of weak measure solutions $\left(u_{t}^{1}\right)_{t \geq 0},\left(u_{t}^{2}\right)_{t \geq 0} \subset \mathcal{P}(\mathcal{J})$ to $(24)$ starting from $u_{0}^{1}$ and $u_{0}^{2}$, i.e., such that for $i=1,2$ and all $t \geq 0$

$$
\int_{0}^{t} \int_{\mathcal{J}} d\left(x_{1}, x_{2}\right) u_{s}^{i}\left(\mathrm{~d} x_{1}, \mathrm{~d} x_{2}\right) \mathrm{d} s<\infty
$$


and for $i=1,2$, all $t \geq 0$ and all $\varphi \in C_{c}^{1}(\mathcal{J})$,

$$
\begin{aligned}
\int_{\mathcal{J}} \varphi\left(x_{1}, x_{2}\right) & u_{t}^{i}\left(\mathrm{~d} x_{1}, \mathrm{~d} x_{2}\right)=\int_{\mathcal{J}} \varphi\left(x_{1}, x_{2}\right) u_{0}^{i}\left(\mathrm{~d} x_{1}, \mathrm{~d} x_{2}\right) \\
& +\int_{0}^{t} \int_{\mathcal{J}}\left[\frac{\partial \varphi\left(x_{1}, x_{2}\right)}{\partial x_{1}}+\frac{\partial \varphi\left(x_{1}, x_{2}\right)}{\partial x_{2}}+d\left(x_{1}, x_{2}\right)\left[\varphi\left(0, x_{1}\right)-\varphi\left(x_{1}, x_{2}\right)\right]\right] u_{s}^{i}\left(\mathrm{~d} x_{1}, \mathrm{~d} x_{2}\right) \mathrm{d} s .
\end{aligned}
$$

Moreover, for all $t \geq 0$, we have

$$
\mathcal{T}_{\varrho}\left(u_{t}^{1}, u_{t}^{2}\right) \leq \mathcal{T}_{\varrho}\left(u_{0}^{1}, u_{0}^{2}\right)
$$

Proof. We assume (25)-(26), consider $u_{0}^{1}, u_{0}^{2} \in \mathcal{P}(\mathcal{J})$ and a coupling $v_{0} \in \mathcal{H}\left(u_{0}^{1}, u_{0}^{2}\right)$. There exists a family $\left(v_{t}\right)_{t \geq 0}$ of probability measures on $\mathcal{J}^{2}$ such that for all $t \geq 0$

$$
\int_{0}^{t} \iint\left[d\left(x_{1}, x_{2}\right)+d\left(\widetilde{x}_{1}, \widetilde{x}_{2}\right)\right] v_{t}\left(\mathrm{~d} x_{1}, \mathrm{~d} x_{2}, \mathrm{~d} \widetilde{x}_{1}, \mathrm{~d} \widetilde{x}_{2}\right)<\infty
$$

and which weakly solves, with zero flux boudary conditions at $x_{1}=0$ and $x_{2}=0$,

$$
\begin{aligned}
\frac{\partial v_{t}}{\partial t}+\frac{\partial v_{t}}{\partial x_{1}}+\frac{\partial v_{t}}{\partial x_{2}}+ & \frac{\partial v_{t}}{\partial \widetilde{x}_{1}}+\frac{\partial v_{t}}{\partial \widetilde{x}_{2}}+\left(d\left(x_{1}, x_{2}\right) \vee d\left(\widetilde{x}_{1}, \widetilde{x}_{2}\right)\right) v_{t} \\
= & \delta\left(x_{1}\right) \delta\left(\widetilde{x}_{1}\right) \iint\left(d\left(x_{2}, z\right) \vee d\left(\widetilde{x}_{2}, \widetilde{z}\right)\right) v_{t}\left(x_{2}, \mathrm{~d} z, \widetilde{x}_{2}, \mathrm{~d} \widetilde{z}\right) \\
& +\delta\left(x_{1}\right) \iint\left(d\left(x_{2}, z\right)-d\left(\widetilde{x}_{1}, \widetilde{x}_{2}\right)\right)_{+} v_{t}\left(x_{2}, \mathrm{~d} z, \widetilde{x}_{1}, \widetilde{x}_{2}\right) \\
& +\delta\left(\widetilde{x}_{1}\right) \iint\left(d\left(x_{1}, x_{2}\right)-d\left(\widetilde{x}_{2}, \widetilde{z}\right)\right)_{+} v_{t}\left(x_{1}, x_{2}, \widetilde{x}_{2}, \mathrm{~d} \widetilde{z}\right) .
\end{aligned}
$$

This means that for all $\varphi \in C_{c}^{1}\left(\mathcal{J}^{2}\right)$, all $t \geq 0$,

$$
\begin{aligned}
& \iint \varphi\left(x_{1}, x_{2}, \widetilde{x}_{1}, \widetilde{x}_{2}\right) v_{t}\left(\mathrm{~d} x_{1}, \mathrm{~d} x_{2}, \mathrm{~d} \widetilde{x}_{1}, \mathrm{~d} \widetilde{x}_{2}\right)=\iint \varphi\left(x_{1}, x_{2}, \widetilde{x}_{1}, \widetilde{x}_{2}\right) v_{0}\left(\mathrm{~d} x_{1}, \mathrm{~d} x_{2}, \mathrm{~d} \widetilde{x}_{1}, \mathrm{~d} \widetilde{x}_{2}\right) \\
&+\int_{0}^{t} \iint\left[\frac{\partial \varphi}{\partial x_{1}}+\frac{\partial \varphi}{\partial x_{2}}+\frac{\partial \varphi}{\partial \widetilde{x}_{1}}+\frac{\partial \varphi}{\partial \widetilde{x}_{2}}\right]\left(x_{1}, x_{2}, \widetilde{x}_{1}, \widetilde{x}_{2}\right) v_{s}(\mathrm{~d} x, \mathrm{~d} z, \mathrm{~d} y, \mathrm{~d} r) \mathrm{d} s \\
&+\int_{0}^{t} \iint\left(d\left(x_{1}, x_{2}\right) \wedge d\left(\widetilde{x}_{1}, \widetilde{x}_{2}\right)\right)\left[\varphi\left(0, x_{1}, 0, \widetilde{x}_{1}\right)-\varphi\left(x_{1}, x_{2}, \widetilde{x}_{1}, \widetilde{x}_{2}\right)\right] \\
&+\left(d\left(x_{1}, x_{2}\right)-d\left(\widetilde{x}_{1}, \widetilde{x}_{2}\right)\right)_{+}\left[\varphi\left(0, x_{1}, \widetilde{x}_{1}, \widetilde{x}_{2}\right)-\varphi\left(x_{1}, x_{2}, \widetilde{x}_{1}, \widetilde{x}_{2}\right)\right] \\
&\left.+\left(d\left(\widetilde{x}_{1}, \widetilde{x}_{2}\right)-d\left(x_{1}, x_{2}\right)\right)_{+}\left[\varphi\left(x_{1}, x_{2}, 0, \widetilde{x}_{1}\right)-\varphi\left(x_{1}, x_{2}, \widetilde{x}_{1}, \widetilde{x}_{2}\right)\right]\right) v_{s}\left(\mathrm{~d} x_{1}, \mathrm{~d} x_{2}, \mathrm{~d} \widetilde{x}_{2}, \mathrm{~d} \widetilde{x}_{2}\right) \mathrm{d} s .
\end{aligned}
$$

Using (29) and (30) with a function $\varphi$ depending only on $\left(x_{1}, x_{2}\right)$ (or $\left(\widetilde{x}_{1}, \widetilde{x}_{2}\right)$ ), we see that the marginals of $v$ satisfy (27) and (28).

The existence for (29)-(30) follows as usual from the following a priori tightness estimate. By the de la Vallée Poussin theorem, there is a function $h:[0, \infty) \rightarrow[0, \infty)$ such that $\lim _{x \rightarrow \infty} h(x)=\infty$ and

$$
C:=\iint\left[h\left(x_{1}\right)+h\left(x_{2}\right)+h\left(\widetilde{x}_{1}\right)+h\left(\widetilde{x}_{2}\right)\right] v_{0}\left(\mathrm{~d} x_{1}, \mathrm{~d} x_{2}, \mathrm{~d} \widetilde{x}_{1}, \mathrm{~d} \widetilde{x}_{2}\right)<\infty .
$$


Choosing moreover $h$ smooth, satisfying $h(0)=0$ and $0 \leq h^{\prime} \leq 1$, applying $(30)$ with $\varphi\left(x_{1}, x_{2}, \widetilde{x}_{1}, \widetilde{x}_{2}\right)=$ $h\left(x_{1}\right)+h\left(x_{2}\right)+h\left(\widetilde{x}_{1}\right)+h\left(\widetilde{x}_{2}\right)$, one easily concludes as usual that for all $t \geq 0$,

$$
\begin{aligned}
& \iint {\left[h\left(x_{1}\right)+h\left(x_{2}\right)+h\left(\widetilde{x}_{1}\right)+h\left(\widetilde{x}_{2}\right)\right] v_{t}\left(\mathrm{~d} x_{1}, \mathrm{~d} x_{2}, \mathrm{~d} \widetilde{x}_{1}, \mathrm{~d} \widetilde{x}_{2}\right) } \\
& \quad+\int_{0}^{t} \iint\left(d\left(x_{1}, x_{2}\right) h\left(x_{2}\right)+d\left(\widetilde{x}_{1}, \widetilde{x}_{2}\right) h\left(\widetilde{x}_{2}\right)\right) v_{s}\left(\mathrm{~d} x_{1}, \mathrm{~d} x_{2}, \mathrm{~d} \widetilde{x}_{1}, \mathrm{~d} \widetilde{x}_{2}\right) \mathrm{d} s \leq C+4 t .
\end{aligned}
$$

Recalling that $h$ increases to infinity and that $h\left(x_{1}\right) \leq h\left(x_{2}\right)$ for all $\left(x_{1}, x_{2}\right) \in \mathcal{J}$, this a priori tightness estimate is enough to prove existence for (29)-(30).

We now apply $(23)$ with $\varphi=\varrho$, where $\varrho\left(x_{1}, x_{2}, \widetilde{x}_{1}, \widetilde{x}_{2}\right)=\min \left(a, 2\left|x_{1}-\widetilde{x}_{1}\right|+\left|x_{2}-\widetilde{x}_{2}\right|\right)$. Since

$$
\frac{\partial \varphi}{\partial x_{1}}+\frac{\partial \varphi}{\partial x_{2}}+\frac{\partial \varphi}{\partial \widetilde{x}_{1}}+\frac{\partial \varphi}{\partial \widetilde{x}_{2}}=0
$$

we find, choosing $v_{0}$ such that $\iint \varrho\left(x_{1}, x_{2}, \widetilde{x}_{1}, \widetilde{x}_{2}\right) v_{0}\left(\mathrm{~d} x_{1}, \mathrm{~d} x_{2}, \mathrm{~d} \widetilde{x}_{1}, \mathrm{~d} \widetilde{x}_{2}\right)=\mathcal{T}_{\rho}\left(u_{0}^{1}, u_{0}^{2}\right)$,

$\iint \varrho\left(x_{1}, x_{2}, \widetilde{x}_{1}, \widetilde{x}_{2}\right) v_{t}\left(\mathrm{~d} x_{1}, \mathrm{~d} x_{2}, \mathrm{~d} \widetilde{x}_{1}, \mathrm{~d} \widetilde{x}_{2}\right) \leq \mathcal{T}_{\rho}\left(u_{0}^{1}, u_{0}^{2}\right)+\int_{0}^{t} \iint \Delta\left(x_{1}, x_{2}, \widetilde{x}_{1}, \widetilde{x}_{2}\right) v_{s}\left(\mathrm{~d} x_{1}, \mathrm{~d} x_{2}, \mathrm{~d} \widetilde{x}_{1}, \mathrm{~d} \widetilde{x}_{2}\right) \mathrm{d} s$

where

$$
\begin{aligned}
\Delta\left(x_{1}, x_{2}, \widetilde{x}_{1}, \widetilde{x}_{2}\right)= & -\left(d\left(x_{1}, x_{2}\right) \vee d\left(\widetilde{x}_{1}, \widetilde{x}_{2}\right)\right)\left(\left[2\left|x_{1}-\widetilde{x}_{1}\right|+\left|x_{2}-\widetilde{x}_{2}\right|\right] \wedge a\right) \\
& +\left(d\left(x_{1}, x_{2}\right) \wedge d\left(\widetilde{x}_{1}, \widetilde{x}_{2}\right)\right)\left(\left|x_{1}-\widetilde{x}_{1}\right| \wedge a\right) \\
& +\left(d\left(x_{1}, x_{2}\right)-d\left(\widetilde{x}_{1}, \widetilde{x}_{2}\right)\right)_{+}\left(\left[2\left|\widetilde{x}_{1}\right|+\left|x_{1}-\widetilde{x}_{2}\right|\right] \wedge a\right) \\
& +\left(d\left(\widetilde{x}_{1}, \widetilde{x}_{2}\right)-d\left(x_{1}, x_{2}\right)\right)_{+}\left(\left[2\left|x_{1}\right|+\left|\widetilde{x}_{1}-x_{2}\right|\right] \wedge a\right) .
\end{aligned}
$$

Since $\mathcal{T}_{\rho}\left(u_{t}^{1}, u_{t}^{2}\right) \leq \iint \rho\left(x_{1}, x_{2}, \widetilde{x}_{1}, \widetilde{x}_{2}\right) v_{t}\left(\mathrm{~d} x_{1}, \mathrm{~d} x_{2}, \mathrm{~d} \widetilde{x}_{1}, \mathrm{~d} \widetilde{x}_{2}\right)$, it only remains to check that $\Delta$ is always non-positive.

When first $2\left|x_{1}-\widetilde{x}_{1}\right|+\left|x_{2}-\widetilde{x}_{2}\right| \geq a$, this is obvious.

When $2\left|x_{1}-\widetilde{x}_{1}\right|+\left|x_{2}-\widetilde{x}_{2}\right| \leq a$, it suffices to verify that

$$
\begin{aligned}
\left(d\left(x_{1}, x_{2}\right) \vee d\left(\widetilde{x}_{1}, \widetilde{x}_{2}\right)\right)\left[2\left|x_{1}-\widetilde{x}_{1}\right|+\left|x_{2}-\widetilde{x}_{2}\right|\right] \\
\geq\left(d\left(x_{1}, x_{2}\right) \wedge d\left(\widetilde{x}_{1}, \widetilde{x}_{2}\right)\right)\left|x_{1}-\widetilde{x}_{1}\right|+\left|d\left(x_{1}, x_{2}\right)-d\left(\widetilde{x}_{1}, \widetilde{x}_{2}\right)\right| a
\end{aligned}
$$

This follows from the fact that

$$
\left(d\left(x_{1}, x_{2}\right) \vee d\left(\widetilde{x}_{1}, \widetilde{x}_{2}\right)\right)\left[\left|x_{1}-\widetilde{x}_{1}\right|+\left|x_{2}-\widetilde{x}_{2}\right|\right] \geq\left|d\left(x_{1}, x_{2}\right)-d\left(\widetilde{x}_{1}, \widetilde{x}_{2}\right)\right| a
$$

by $(26)$.

\section{Growth-fragmentation}

The growth-fragmentation equation arises in several areas of biology. The variable represents for instance the size of cells or the length of biopolymers. It also arises in communication science for TCP 
connections. A large literature is available on the subject and we refer for instance to $[24,12,27,4]$. The model combines growth with a rate $g$ and fragmentation with a rate $d$ and it is written

$$
\begin{cases}\frac{\partial u_{t}(x)}{\partial t}+\frac{\partial\left[g(x) u_{t}(x)\right]}{\partial x}+d(x) u_{t}(x)=\int_{x}^{\infty} d\left(x^{\prime}\right) \kappa\left(x, x^{\prime}\right) u_{t}\left(\mathrm{~d} x^{\prime}\right), & t \geq 0, x \geq 0, \\ u_{t}(x=0)=0, & t \geq 0 .\end{cases}
$$

Usual conditions on the fragmentation kernel are expressed through the identities

$$
\kappa\left(x, x^{\prime}\right)=0 \text { for } x>x^{\prime}, \quad \int_{0}^{x^{\prime}} \kappa\left(x, x^{\prime}\right) \mathrm{d} x=1
$$

which lead to the conservation law

$$
\int_{0}^{\infty} u_{t}(x) \mathrm{d} x=\int_{0}^{\infty} u_{0}(x) \mathrm{d} x=1
$$

To go further, we can specify

$$
\kappa\left(x, x^{\prime}\right)=\frac{1}{x^{\prime}} \beta\left(\frac{x}{x^{\prime}}\right) \quad \text { for some } \beta \in \mathcal{P}([0,1]),
$$

see the end of the section for a more general possible setting. We then assume that

$$
g, d \in C([0, \infty)), \quad g \text { is non-increasing }, \quad g(0) \geq 0, \quad d \geq 0,
$$

and

$$
\exists a>0 \quad \text { such that } \quad a \leq\left(1-\int_{0}^{1} r \beta(\mathrm{d} r)\right) \inf _{\mid x-y] \leq a} \frac{|x-y| \max (d(x), d(y))}{|d(x)-d(y)|} .
$$

If $\beta$ is non-trivial in that $\int_{0}^{1} r \beta(\mathrm{d} r) \in[0,1)$, then this assumption is verified if e.g. $d(x)=\alpha+\beta x^{p}$, provided $\alpha>0, \beta \geq 0$ and $p \geq 1$.

Theorem 5 We assume (33)-(34)-(35). We choose again, on $[0, \infty) \times[0, \infty)$ the cost

$$
\varrho(x, y)=\min (a,|x-y|) .
$$

For any $u_{0}^{1}, u_{0}^{2} \in \mathcal{P}([0, \infty))$, there exists a pair of weak measure solutions $\left(u_{t}^{1}\right)_{t \geq 0},\left(u_{t}^{2}\right)_{t \geq 0} \subset \mathcal{P}([0, \infty))$ to (31), starting from $u_{0}^{1}$ and $u_{0}^{2}$, i.e., such that for $i=1,2$, for all $t \geq 0$, all $\bar{A} \geq 1$,

$$
\int_{0}^{t} \int_{A+1}^{\infty} d(x) \int_{0}^{A / x} \beta(\mathrm{d} r) u_{s}^{i}(\mathrm{~d} x) \mathrm{d} s<\infty
$$

and for all $t \geq 0$, all $\varphi \in C_{c}^{1}([0, \infty))$,

$$
\int_{0}^{\infty} \varphi(x) u_{t}^{i}(\mathrm{~d} x)=\int_{0}^{\infty} \varphi(x) u_{0}^{i}(\mathrm{~d} x)+\int_{0}^{t} \int_{0}^{\infty}\left[g(x) \varphi^{\prime}(x)+d(x) \int_{0}^{1}(\varphi(r x)-\varphi(x)) \beta(\mathrm{d} r)\right] u_{s}^{i}(\mathrm{~d} x) \mathrm{d} s .
$$

Moreover, for all $t \geq 0$, we have

$$
\mathcal{T}_{\varrho}\left(u_{t}^{1}, u_{t}^{2}\right) \leq \mathcal{T}_{\varrho}\left(u_{0}^{1}, u_{0}^{2}\right) .
$$


Observe that (37) makes sense thanks to (36): for $\varphi \in C_{c}^{1}([0, \infty))$ supported in $[0, A]$,

$$
\left|d(x) \int_{0}^{1}(\varphi(r x)-\varphi(x)) \beta(\mathrm{d} r)\right| \leq \mathbb{I}_{\{x \leq A+1\}} 2\|\varphi\|_{\infty} \sup _{[0, A+1]} d+\mathbb{I}_{\{x>A+1\}}\|\varphi\|_{\infty} d(x) \int_{0}^{A / x} \beta(\mathrm{d} r),
$$

where we used a rough upper bound by $A+1$ to fit our assumption.

Proof. Consider $u_{0}^{1}, u_{0}^{2} \in \mathcal{P}([0, \infty))$ and a coupling $v_{0} \in \mathcal{H}\left(u_{0}^{1}, u_{0}^{2}\right)$. There exists a family $\left(v_{t}\right)_{t \geq 0}$ of probability measures on $[0, \infty)^{2}$ satisfying, for all $t \geq 0$, all $A \geq 1$,

$$
\int_{0}^{t} \iint\left[\mathbb{I}_{\{x \geq A+1\}} d(x) \int_{0}^{A / x} \beta(\mathrm{d} r)+\mathbb{1}_{\{y \geq A+1\}} d(y) \int_{0}^{A / y} \beta(\mathrm{d} r)\right] v_{s}(\mathrm{~d} x, \mathrm{~d} y) \mathrm{d} s<\infty
$$

and solving weakly

$$
\begin{aligned}
& \frac{\partial v_{t}}{\partial t}+\frac{\partial}{\partial x}\left[g(x) v_{t}\right]+\frac{\partial}{\partial y}\left[g(y) v_{t}\right]+(d(x) \vee d(y)) v_{t}= \\
& \quad \iint\left(d\left(x^{\prime}\right) \wedge d\left(y^{\prime}\right)\right) \frac{1}{x^{\prime} y^{\prime}} \beta\left(\frac{x}{x^{\prime}}\right) \delta\left(\frac{x}{x^{\prime}}-\frac{y}{y^{\prime}}\right) \beta\left(\frac{x}{x^{\prime}}\right) v_{t}\left(\mathrm{~d} x^{\prime}, \mathrm{d} y^{\prime}\right) \\
& \quad+\int\left(d\left(x^{\prime}\right)-d(y)\right)_{+} \frac{1}{x^{\prime}} \beta\left(\frac{x}{x^{\prime}}\right) v_{t}\left(\mathrm{~d} x^{\prime}, y\right)+\int\left(d\left(y^{\prime}\right)-d(x)\right)_{+} \frac{1}{y^{\prime}} \beta\left(\frac{y}{y^{\prime}}\right) v\left(x, \mathrm{~d} y^{\prime}\right) .
\end{aligned}
$$

This means that for all $t \geq 0$, all $\varphi \in C_{c}^{1}\left([0, \infty)^{2}\right)$,

$$
\begin{aligned}
& \iint \varphi(x, y) v_{t}(\mathrm{~d} x, \mathrm{~d} y)=\iint \varphi(x, y) v_{0}(\mathrm{~d} x, \mathrm{~d} y)+\int_{0}^{t} \iint\left[g(x) \frac{\partial \varphi(x, y)}{\partial x}+g(y) \frac{\partial \varphi(x, y)}{\partial y}\right] v_{s}(\mathrm{~d} x, \mathrm{~d} y) \mathrm{d} s \\
& \quad+\int_{0}^{t} \iint(d(x) \wedge d(y)) \int_{0}^{1}[\varphi(r x, r y)-\varphi(x, y)] \beta(\mathrm{d} r) v_{s}(\mathrm{~d} x, \mathrm{~d} y) \mathrm{d} s \\
& \quad+\int_{0}^{t} \iint(d(x)-d(y))_{+} \int_{0}^{1}[\varphi(r x, y)-\varphi(x, y)] \beta(\mathrm{d} r) v_{s}(\mathrm{~d} x, \mathrm{~d} y) \mathrm{d} s \\
& \quad+\int_{0}^{t} \iint(d(y)-d(x))_{+} \int_{0}^{1}[\varphi(x, r y)-\varphi(x, y)] \beta(\mathrm{d} r) v_{s}(\mathrm{~d} x, \mathrm{~d} y) \mathrm{d} s
\end{aligned}
$$

One checks as usual that the two marginals of $v_{t}$ solve (36)-(37). Next, as in the previous sections, one finds that there is a function $h:[0, \infty) \rightarrow[0, \infty)$, strictly increasing to infinity, which taken as a test function gives

$$
\iint[h(x)+h(y)] v_{t}(\mathrm{~d} x, \mathrm{~d} y)+\int_{0}^{t} \iint[d(x) H(x)+d(\widetilde{x}) H(\widetilde{x})] v_{s}(\mathrm{~d} x, \mathrm{~d} y) \mathrm{d} s \leq C+2 \bar{C} t,
$$

where $H(x)=\int_{0}^{1}(h(x)-h(r x)) \beta(\mathrm{d} r)$ and $\bar{C}=\sup _{x \geq 0} g(x) h^{\prime}(x)$. This a priori estimate is sufficient to prove tightness and construct a solution to (36)-(37), because for any $A \geq 1$, any $x \geq A+1$, one has

$$
\int_{0}^{A / x} \beta(\mathrm{d} r) \leq \int_{0}^{A / x} \frac{h(x)-h(r x)}{h(A+1)-h(A)} \beta(\mathrm{d} r) \leq \frac{H(x)}{h(A+1)-h(A)} .
$$

Applying now the above equation to $\varphi=\varrho$, where $\varrho(x, y)=\min (a,|x-y|)$, one finds as usual, if choosing $v_{0}$ correctly and using that $g$ is non-increasing, that

$$
\iint \varrho(x, y) v_{t}(\mathrm{~d} x, \mathrm{~d} y) \leq \mathcal{T}_{\varrho}\left(u_{0}^{1}, u_{0}^{2}\right)+\int_{0}^{t} \iint \Delta(x, y) v_{s}(\mathrm{~d} x, \mathrm{~d} y) \mathrm{d} s,
$$


where

$$
\begin{aligned}
\Delta(x, y)= & -(d(x) \vee d(y)) \varrho(x, y)+(d(x) \wedge d(y)) \int_{0}^{1} \varrho(r x, r y) \beta(\mathrm{d} r) \\
& +(d(x)-d(y))_{+} \int_{0}^{1} \varrho(r x, y) \beta(\mathrm{d} r)+(d(y)-d(x))_{+} \int_{0}^{1} \varrho(x, r y) \beta(\mathrm{d} r) .
\end{aligned}
$$

We finally show that $\Delta$ is alway non-positive. If first $|x-y| \geq a$, it is enough to check that

$$
a(d(x) \vee d(y)) \geq a(d(x) \wedge d(y))+a(d(x)-d(y))_{+}+a(d(y)-d(x))_{+},
$$

which is obvious. If next $|x-y| \leq a$, it suffices to check that

$$
(d(x) \vee d(y))|x-y| \geq(d(x) \wedge d(y))\left[\int_{0}^{1} r \beta(\mathrm{d} r)\right]|x-y|+a(d(x)-d(y))_{+}+a(d(y)-d(x))_{+},
$$

which follows from the fact that

$$
\left[1-\int_{0}^{1} r \beta(\mathrm{d} r)\right](d(x) \vee d(y))|x-y| \geq a|d(x)-d(y)|
$$

thanks to (35).

A little study shows that the result still holds true if assuming, instead of (33), that the family of fragmentation kernels $(\kappa(\cdot, x))_{x \in \mathbb{R}_{+}} \subset \mathcal{P}\left(\mathbb{R}_{+}\right)$satisfies $\kappa([0, x], x)=1$ for all $x \geq 0$ and

$$
\exists m \in[0,1), \quad \text { for all } x, y \in \mathbb{R}_{+}, \quad W_{1}(\kappa(\cdot, x), \kappa(\cdot, y)) \leq m|x-y|,
$$

where $W_{1}$ is the usual Monge-Kantorovich distance on $\mathcal{P}\left(\mathbb{R}_{+}\right)$, and replacing (35) by

$$
\exists a>0 \quad \text { such that } \quad a \leq(1-m) \inf _{\mid x-y] \leq a} \frac{|x-y| \max (d(x), d(y))}{|d(x)-d(y)|} .
$$

Indeed, it suffices to apply the usual strategy, starting from the coupling equation :

$$
\begin{gathered}
\iint \varphi(x, y) v_{t}(\mathrm{~d} x, \mathrm{~d} y)=\iint \varphi(x, y) v_{0}(\mathrm{~d} x, \mathrm{~d} y)+\int_{0}^{t} \iint\left[g(x) \frac{\partial \varphi(x, y)}{\partial x}+g(y) \frac{\partial \varphi(x, y)}{\partial y}\right] v_{s}(\mathrm{~d} x, \mathrm{~d} y) \mathrm{d} s \\
+\int_{0}^{t} \iint(d(x) \wedge d(y)) \int_{0}^{x} \int_{0}^{y}\left[\varphi\left(x^{\prime}, y^{\prime}\right)-\varphi(x, y)\right] \bar{\kappa}\left(\mathrm{d} x^{\prime}, \mathrm{d} y^{\prime}, x, y\right) v_{s}(\mathrm{~d} x, \mathrm{~d} y) \mathrm{d} s \\
+\int_{0}^{t} \iint(d(x)-d(y))_{+} \int_{0}^{x}\left[\varphi\left(x^{\prime}, y\right)-\varphi(x, y)\right] \kappa\left(\mathrm{d} x^{\prime}, x\right) v_{s}(\mathrm{~d} x, \mathrm{~d} y) \mathrm{d} s \\
+\int_{0}^{t} \iint(d(y)-d(x))_{+} \int_{0}^{y}\left[\varphi\left(x, y^{\prime}\right)-\varphi(x, y)\right] \kappa\left(\mathrm{d} y^{\prime}, y\right) v_{s}(\mathrm{~d} x, \mathrm{~d} y) \mathrm{d} s
\end{gathered}
$$

where for each $x, y \in \mathbb{R}_{+}, \bar{\kappa}(\cdot, \cdot, x, y) \in \mathcal{H}(\kappa(\cdot, x), \kappa(\cdot, y))$ satisfies

$$
\iint\left|x^{\prime}-y^{\prime}\right| \bar{\kappa}\left(\mathrm{d} x^{\prime}, \mathrm{d} y^{\prime}, x, y\right)=W_{1}(\kappa(\cdot, x), \kappa(\cdot, y)) .
$$




\section{$6 \quad$ Age and size structure}

Models have been proposed which use several stucture variables. For instance, age or size only are not enough to predict cell division. But a combination of both (or other physiological variables as size increment) have been used, see $[15,16]$, leading to write

$$
\begin{cases}\frac{\partial u_{t}(x, z)}{\partial t}+\frac{\partial u_{t}(x, z)}{\partial x}+\frac{\partial\left[g(z) u_{t}(x, z)\right]}{\partial z}+d(x, z) u_{t}(x, z)=0, & t \geq 0, x>0, z>0, \\ u_{t}(x, z=0)=0, & t \geq 0, x>0, \\ u_{t}(x=0, z)=\int_{x=0}^{\infty} \int_{z^{\prime}=z}^{\infty} d\left(x^{\prime}, z^{\prime}\right) \kappa\left(z, z^{\prime}\right) u_{t}\left(\mathrm{~d} x^{\prime}, \mathrm{d} z^{\prime}\right), & t \geq 0, z>0\end{cases}
$$

The state space is $\mathcal{J}=[0, \infty)^{2}$. We assume that the coagulation kernel has the specific form (33), that

$$
g \in C([0, \infty)), \quad g \text { is non-increasing, } \quad g(0) \geq 0, \quad d \in C(\mathcal{J}), \quad d \geq 0
$$

and that

$$
\exists a>0 \quad \text { such that } a \leq\left(1-\int_{0}^{1} r \beta(\mathrm{d} r)\right) \inf _{|x-z|+|\widetilde{x}-\widetilde{z}| \leq a} \frac{|x-\widetilde{x}|+|z-\tilde{z}|}{|d(x, z)-d(\widetilde{x}, \widetilde{z})|} \max (d(x, z), d(\widetilde{x}, \widetilde{z})) .
$$

Theorem 6 Assume (33)-(42)-(43) and consider the cost

$$
\varrho(x, z, \widetilde{x}, \widetilde{z})=\min (a,|x-\widetilde{x}|+|z-\tilde{z}|) .
$$

For any $u_{0}^{1}, u_{0}^{2} \in \mathcal{P}(\mathcal{J})$, there exists a pair of weak measure solutions $\left(u_{t}^{1}\right)_{t \geq 0},\left(u_{t}^{2}\right)_{t \geq 0} \subset \mathcal{P}(\mathcal{J})$ to $(41)$, starting from $u_{0}^{1}$ and $u_{0}^{2}$, i.e., such that for $i=1,2$ and all $t \geq 0$, all $A \geq 1$,

$$
\int_{0}^{t} \int_{\mathcal{J}} d(x, z)\left[\mathbb{I}_{\{x \geq A+1\}}+\mathbb{I}_{\{x \leq A+1, z \geq A+1\}} \int_{0}^{A / z} \beta(\mathrm{d} r)\right] u_{s}^{i}(\mathrm{~d} x, \mathrm{~d} z) \mathrm{d} s<\infty
$$

and for all $t \geq 0$, all $\varphi \in C_{c}^{1}(\mathcal{J})$,

$$
\begin{aligned}
& \int_{\mathcal{J}} \varphi(x, z) u_{t}^{i}(\mathrm{~d} x, \mathrm{~d} z)=\int_{\mathcal{J}} \varphi(x, z) u_{0}^{i}(\mathrm{~d} x, \mathrm{~d} z) \\
& \quad+\int_{0}^{t} \int_{\mathcal{J}}\left[\frac{\partial \varphi(x, z)}{\partial x}+g(z) \frac{\partial \varphi(x, z)}{\partial z}+d(x, z) \int_{0}^{1}(\varphi(0, r z)-\varphi(x, z)) \beta(\mathrm{d} r)\right] u_{s}^{i}(\mathrm{~d} x, \mathrm{~d} z) \mathrm{d} s .
\end{aligned}
$$

Moreover, for all $t \geq 0$, we have

$$
\mathcal{T}_{\varrho}\left(u_{t}^{1}, u_{t}^{2}\right) \leq \mathcal{T}_{\varrho}\left(u_{0}^{1}, u_{0}^{2}\right)
$$

Here again, (45) makes sense thanks to (44): if $\varphi \in C_{c}(\mathcal{J})$ is supported in $[0, A]^{2}$, then

$$
\begin{aligned}
d(x, z)\left|\int_{0}^{1}(\varphi(0, r z)-\varphi(x, z)) \beta(\mathrm{d} r)\right| \leq & \mathbb{I}_{\{x \leq A+1, z \leq A+1\}} 2\|\varphi\|_{\infty} \sup _{[0, A+]^{2}} d+\mathbb{I}_{\{x \geq A+1\}}\|\varphi\|_{\infty} d(x, z) \\
& +\mathbb{I}_{\{x \leq A+1, z \geq A+1\}}\|\varphi\|_{\infty} d(x, z) \int_{0}^{A / z} \beta(\mathrm{d} r) .
\end{aligned}
$$


Proof. As before, we consider $u_{0}^{1}, u_{0}^{2} \in \mathcal{P}(\mathcal{J})$ and a coupling $v_{0} \in \mathcal{H}\left(u_{0}^{1}, u_{0}^{2}\right)$. There exists a family $\left(v_{t}\right)_{t \geq 0}$ of probability measures on $\mathcal{J}^{2}$ such that, for all $t \geq 0$,

$$
\begin{aligned}
\int_{0}^{t} \int_{\mathcal{J}}\left(d(x, z)\left[\mathbb{I}_{\{x \geq A+1\}}+\mathbb{I}_{\{x \leq A+1, z \geq A+1\}} \int_{0}^{A / z} \beta(\mathrm{d} r)\right]\right. \\
\left.\quad+d(\widetilde{x}, \widetilde{z})\left[\mathbb{I}_{\{\widetilde{x} \geq A+1\}}+\mathbb{I}_{\{\widetilde{x} \leq A+1, \widetilde{z} \geq A+1\}} \int_{0}^{A / \widetilde{z}} \beta(\mathrm{d} r)\right]\right) v_{s}(\mathrm{~d} x, \mathrm{~d} z, \mathrm{~d} \widetilde{x}, \mathrm{~d} \widetilde{z}) \mathrm{d} s<\infty
\end{aligned}
$$

and that weakly solves

$$
\begin{aligned}
\frac{\partial}{\partial t} v_{t}(x, z, \widetilde{x}, \widetilde{z})+ & \frac{\partial v_{t}}{\partial x}+\frac{\partial v_{t}}{\partial \widetilde{x}}+\frac{\partial}{\partial z}\left[g(z) v_{t}\right]+\frac{\partial v_{t}}{\partial \widetilde{z}}\left[g(\widetilde{z}) v_{t}\right]+b(x, z) \vee d(\widetilde{x}, \widetilde{z}) v_{t} \\
= & \delta(x) \delta(\widetilde{x}) \iint\left(d\left(x^{\prime}, z^{\prime}\right) \wedge d\left(\widetilde{x}^{\prime}, \widetilde{z}^{\prime}\right)\right) \frac{1}{z^{\prime} \widetilde{z}^{\prime}} \beta\left(\frac{z}{z^{\prime}}\right) \delta\left(\frac{z}{z^{\prime}}-\frac{\widetilde{z}}{\widetilde{z}^{\prime}}\right) v_{t}\left(\mathrm{~d} x^{\prime}, \mathrm{d} z^{\prime}, \mathrm{d} \widetilde{x}^{\prime}, \mathrm{d} \widetilde{z}^{\prime}\right) \\
& +\delta(x) \int\left(d\left(x^{\prime}, z^{\prime}\right)-d(\widetilde{x}, \widetilde{z})\right)_{+} \frac{1}{z^{\prime}} \beta\left(\frac{z}{z^{\prime}}\right) v_{t}\left(\mathrm{~d} x^{\prime}, \mathrm{d} z^{\prime}, \widetilde{x}, \widetilde{z}\right) \\
& +\delta(\widetilde{x}) \int\left(d\left(\widetilde{x}^{\prime}, \widetilde{z}^{\prime}\right)-d(x, z)\right)_{+} \frac{1}{\widetilde{z}^{\prime}} \beta\left(\frac{\widetilde{z}}{\widetilde{z}^{\prime}}\right) v_{t}\left(x, z, \mathrm{~d} \widetilde{x}^{\prime}, \mathrm{d} \widetilde{z}^{\prime}\right)
\end{aligned}
$$

This equation means that for all $t \geq 0$, for all $\varphi \in C_{c}^{1}\left(\mathcal{J}^{2}\right)$,

$$
\begin{gathered}
\iint \varphi(x, z, \widetilde{x}, \widetilde{z}) v_{t}(\mathrm{~d} x, \mathrm{~d} z, d \widetilde{x}, d \widetilde{z})=\iint \varphi(x, z, \widetilde{x}, \widetilde{z}) v_{0}(\mathrm{~d} x, \mathrm{~d} z, d \widetilde{x}, d \widetilde{z}) \\
+\int_{0}^{t} \iint\left[\frac{\partial \varphi}{\partial x}+\frac{\partial \varphi}{\partial \widetilde{x}}+g(z) \frac{\partial \varphi}{\partial z}+g(\widetilde{z}) \frac{\partial \varphi}{\partial \widetilde{z}}\right](x, z, \widetilde{x}, \widetilde{z}) v_{s}(\mathrm{~d} x, \mathrm{~d} z, d \widetilde{x}, d \widetilde{z}) \mathrm{d} s \\
+\int_{0}^{t} \iint\left[(d(x, z) \wedge d(\widetilde{x}, \widetilde{z})) \int_{0}^{1}[\varphi(0, r z, 0, r \widetilde{z})-\varphi(x, z, \widetilde{x}, \widetilde{z})] \beta(\mathrm{d} r)\right. \\
+(d(x, z)-d(\widetilde{x}, \widetilde{z}))_{+} \int_{0}^{1}[\varphi(0, r z, \widetilde{x}, \widetilde{z})-\varphi(x, z, \widetilde{x}, \widetilde{z})] \beta(\mathrm{d} r) \\
\left.+(d(\widetilde{x}, \widetilde{z})-d(x, z))_{+} \int_{0}^{1}[\varphi(x, z, 0, r \widetilde{z})-\varphi(x, z, \widetilde{x}, \widetilde{z})] \beta(\mathrm{d} r)\right] v_{s}(\mathrm{~d} x, \mathrm{~d} z, d \widetilde{x}, d \widetilde{z}) \mathrm{d} s .
\end{gathered}
$$

The two marginals of $v_{t}$ solve (44)-(45). Next, one finds as usual that there is a function $h:[0, \infty) \rightarrow$ $[0, \infty)$, strictly increasing to infinity, such that

$$
\begin{aligned}
& \iinth(x)+h(z)+h(\widetilde{x})+h(\widetilde{z})] v_{t}(\mathrm{~d} x, \mathrm{~d} z, \mathrm{~d} \widetilde{x}, \mathrm{~d} \widetilde{z}) \\
& \quad+\int_{0}^{t} \iint[d(x, z) H(x, z)+d(\widetilde{x}, \widetilde{z}) H(\widetilde{x}, \widetilde{z})] v_{s}(\mathrm{~d} x, \mathrm{~d} z, \mathrm{~d} \widetilde{x}, \mathrm{~d} \widetilde{z}) \mathrm{d} s \leq C+2(1+\bar{C}) t,
\end{aligned}
$$

where $H(x, z)=h(x)+\int_{0}^{1}(h(x)-h(r x)) \beta(\mathrm{d} r)$ and $\bar{C}=\sup _{x \geq 0} g(x) h^{\prime}(x)$. This a priori estimate is sufficient to construct a solution to (46)-(47), because for any $\bar{A} \geq 1$

$$
\mathbb{1}_{\{x \geq A+1\}}+\mathbb{I}_{\{x \leq A+1, z \geq A+1\}} \int_{0}^{A / z} \beta(\mathrm{d} r) \leq \frac{h(x)}{h(A+1)}+\int_{0}^{1} \frac{h(x)-h(r x)}{h(A+1)-h(A)} \beta(\mathrm{d} r) \leq \frac{H(x)}{h(A+1)-h(A)} .
$$


Following the usual procedure to prove the decay property, that we do not repeat again, it suffices, to conclude the proof, to check that

$$
\begin{aligned}
(d(x, z) & \vee d(\widetilde{x}, \widetilde{z})) \min (a,|x-\widetilde{x}|+|z-\tilde{z}|) \\
& \geq(d(x, z) \wedge d(\widetilde{x}, \widetilde{z})) \int_{0}^{1} \min (a, r|z-\widetilde{z}|) \beta(\mathrm{d} r)+a|d(x, z)-d(\widetilde{x}, \widetilde{z})| .
\end{aligned}
$$

When the min on the left hand side is achieved by $a$ the inequality is obvious. Otherwise, we have to check that

$$
(d(x, z) \vee d(\widetilde{x}, \widetilde{z}))[|x-\widetilde{x}|+|z-\tilde{z}|] \geq \int_{0}^{1} r \beta(\mathrm{d} r)(d(x, z) \wedge d(\widetilde{x}, \widetilde{z}))|z-\widetilde{z}|+a|d(x, z)-d(\widetilde{x}, \widetilde{z})| .
$$

This again is satisfied if

$$
\left(1-\int_{0}^{1} r \beta(\mathrm{d} r)\right)(d(x, z) \vee d(\widetilde{x}, \widetilde{z}))[|x-\widetilde{x}|+|z-\tilde{z}|] \geq a|d(x, z)-d(\widetilde{x}, \widetilde{z})|
$$

which is the condition (43), recall that we are in the case where $|x-\widetilde{x}|+|z-\tilde{z}| \leq a$.

\section{$7 \quad$ Sexually structured populations}

Here a female of type $x^{\prime}$ mates with a male of type $x_{*}^{\prime}$, chosen with the probability $u_{t}(\cdot)$, the newborn is distributed with type $x$ according to the law $K\left(x ; x^{\prime}, x_{*}^{\prime}\right)$. As often in this theory, we assume the distribution of males and females are identical, and rely on the formalism which can be found in $[7,25,9]$ for instance. The (homogeneous) model reads

$$
\partial_{t} u_{t}(x)+u_{t}(x)=\iint_{\mathbb{R}^{2 d}} K\left(x ; x^{\prime}, x_{*}^{\prime}\right) u_{t}\left(\mathrm{~d} x^{\prime}\right) u_{t}\left(\mathrm{~d} x_{*}^{\prime}\right), \quad t \geq 0, x \in \mathbb{R}^{d} .
$$

For keeping the total population constant, the kernel $K \geq 0$ satisfies

$$
\iint_{\mathbb{R}^{d}} K\left(\mathrm{~d} x ; x^{\prime}, x_{*}^{\prime}\right)=1
$$

For instance, we can think of two extreme cases of either a Dirac concentration or a uniform distribution,

$$
K\left(x ; x^{\prime}, x_{*}^{\prime}\right)=\delta_{\theta x^{\prime}+(1-\theta) x_{*}^{\prime}}(x), \quad \theta \in(0,1), \quad \text { or } \quad K\left(x ; x^{\prime}, x_{*}^{\prime}\right)=\frac{1}{\left|x^{\prime}-x_{*}^{\prime}\right|} \mathbb{I}_{\left\{x \in\left(x^{\prime}, x_{*}^{\prime}\right)\right\}} .
$$

These distributions can be generalized to the form

$$
K\left(\mathrm{~d} x ; x^{\prime}, x_{*}^{\prime}\right)=\int_{0}^{1} \delta_{x^{\prime} \sigma+x_{*}^{\prime}(1-\sigma)}(x) h(\mathrm{~d} \sigma)
$$

with $h$ a probability distribution on $[0,1]$ such that $\int_{0}^{1} \sigma h(\mathrm{~d} \sigma)=\theta \in(0,1)$, which is the form we use in the sequel. 
Theorem 7 With the notations and assumptions above, we choose, for some $p \geq 1$,

$$
\varrho(x, y)=|x-y|^{p} .
$$

For any $u_{0}^{1}, u_{0}^{2} \in \mathcal{P}_{p}\left(\mathbb{R}^{d}\right)$, there exists a pair of weak measure solutions $\left(u_{t}^{1}\right)_{t \geq 0},\left(u_{t}^{2}\right)_{t \geq 0} \subset \mathcal{P}_{p}\left(\mathbb{R}^{d}\right)$ to (48), starting from $u_{0}^{1}$ and $u_{0}^{2}$, i.e., such that for $i=1,2$, all $t \geq 0$ and all $\varphi \in C_{c}^{1}\left(\mathbb{R}^{d}\right)$,

$$
\begin{aligned}
& \int_{\mathbb{R}^{d}} \varphi(x) u_{t}^{i}(\mathrm{~d} x)=\int_{\mathbb{R}^{d}} \varphi(x) u_{0}^{i}(\mathrm{~d} x) \\
& \quad+\int_{0}^{t} \iiint\left[\varphi(x)-\theta \varphi\left(x^{\prime}\right)-(1-\theta) \varphi\left(x_{*}^{\prime}\right)\right] K\left(\mathrm{~d} x ; x^{\prime}, x_{*}^{\prime}\right) u_{s}^{i}\left(\mathrm{~d} x^{\prime}, \mathrm{d} x_{*}^{\prime}\right) \mathrm{d} s .
\end{aligned}
$$

Moreover, for all $t \geq 0$, we have

$$
\mathcal{T}_{\varrho}\left(u_{t}^{1}, u_{t}^{2}\right) \leq \mathcal{T}_{\varrho}\left(u_{0}^{1}, u_{0}^{2}\right)
$$

Proof. We use a coupling $\bar{K}\left(x, y ; x^{\prime}, x_{*}^{\prime}, y^{\prime}, y_{*}^{\prime}\right)$, to be chosen later, with the property

$$
\int_{\mathbb{R}^{d}} \bar{K}\left(x, \mathrm{~d} y ; x^{\prime}, x_{*}^{\prime}, y^{\prime}, y_{*}^{\prime}\right)=K\left(x ; x^{\prime}, x_{*}^{\prime}\right), \quad \int_{\mathbb{R}^{d}} \bar{K}\left(\mathrm{~d} x, y ; x^{\prime}, x_{*}^{\prime}, y^{\prime}, y_{*}^{\prime}\right)=K\left(y ; y^{\prime}, y_{*}^{\prime}\right) .
$$

Then, we introduce the coupling equation

$$
\partial_{t} v_{t}(x, y)+v_{t}(x, y)=\iiint \int \bar{K}\left(x, y ; x^{\prime}, x_{*}^{\prime}, y^{\prime}, y_{*}^{\prime}\right) v_{t}\left(\mathrm{~d} x^{\prime}, \mathrm{d} y^{\prime}\right) v_{t}\left(\mathrm{~d} x_{*}^{\prime}, \mathrm{d} y_{*}^{\prime}\right) .
$$

This means that for all $t \geq 0$, for all $\varphi \in C_{c}^{1}\left(\mathbb{R}^{2 d}\right)$,

$$
\begin{array}{r}
\int_{\mathbb{R}^{d}} \varphi(x, y) v_{t}(\mathrm{~d} x, \mathrm{~d} y)=\int_{\mathbb{R}^{d}} \varphi(x, y) v_{0}(\mathrm{~d} x, \mathrm{~d} y)+\int_{0}^{t} \iiint \int\left[\varphi(x, y)-\theta \varphi\left(x^{\prime}, y^{\prime}\right)-(1-\theta) \varphi\left(x_{*}^{\prime}, y_{*}^{\prime}\right)\right] \\
\bar{K}\left(\mathrm{~d} x, \mathrm{~d} y ; x^{\prime}, x_{*}^{\prime}, y^{\prime}, y_{*}^{\prime}\right) v_{s}\left(\mathrm{~d} x^{\prime}, \mathrm{d} y^{\prime}\right) v_{s}\left(\mathrm{~d} x_{*}^{\prime}, \mathrm{d} y_{*}^{\prime}\right) \mathrm{d} s
\end{array}
$$

where $\theta \in(0,1)$ is the same as in (50). For existence, we have to check the tightness in $\mathcal{P}_{p}\left(\mathbb{R}^{d}\right)$. By the de la Vallée Poussin theorem, there is a function $h: \mathbb{R}^{d} \rightarrow[0, \infty)$ such that $\lim _{|x| \rightarrow \infty} h(x)=\infty$ and

$$
\bar{C}:=\iint\left[h(x)|x|^{p}+h(y)|y|^{p}\right] v_{0}(\mathrm{~d} x, \mathrm{~d} y)<\infty .
$$

One can moreover choose $h$ smooth, satisfying $h(0)=0$ and such that $x \mapsto h(x)|x|^{p}$ convex. Choosing $h(x)|x|^{p}+h(y)|y|^{p}$ as a test function in (52), we conclude the bound, for all $t \geq 0$,

$$
\iint\left[h(x)|x|^{p}+h(y)|y|^{p}\right] v_{t}(\mathrm{~d} x, \mathrm{~d} y) \leq \bar{C},
$$

because, by convexity, the second term in the right hand side of (52) is non-positive.

For the non-expansion property, we just have to show that the right hand side is non-positive for $\varrho(x, y)=|x-y|^{p}$ (arguing again after truncation, regularization), if choosing as coupling kernel

$$
\bar{K}\left(\mathrm{~d} x, \mathrm{~d} y ; x^{\prime}, x_{*}^{\prime}, y^{\prime}, y_{*}^{\prime}\right)=\int_{0}^{1} h(\mathrm{~d} \sigma) \delta_{\sigma x^{\prime}+(1-\sigma) x_{*}^{\prime}}(x) \delta_{\sigma y^{\prime}+(1-\sigma) y_{*}^{\prime}}(y) .
$$


The duality formula raises the condition

$$
\int_{0}^{1}\left|\sigma x^{\prime}+(1-\sigma) x_{*}^{\prime}-\sigma y^{\prime}-(1-\sigma) y_{*}^{\prime}\right|^{p} h(\mathrm{~d} \sigma) \leq \theta\left|x^{\prime}-y^{\prime}\right|^{p}+(1-\theta)\left|x_{*}^{\prime}-y_{*}^{\prime}\right|^{p}
$$

which, by convexity, is immediate.

\section{A Uniqueness of measure solutions}

The coupling method is most powerful when the measure solutions are unique. This uniqueness problem, in particular for coefficients with low regularity, can lead to several deep developments, $[2,12]$. Here, we consider regular coefficients so that the Hilbert Uniqueness Method can be applied without difficulty both to the Structured Equations under consideration and to the coupled equations. We treat in details the example of the renewal equation, i.e., (2) when $b=\delta$. We assume that

$$
d \in C([0, \infty)), \quad g(x) \in C_{b}^{1}\left(\mathbb{R}^{+}\right), \quad g(0) \geq 0,
$$

We define the weak solutions (or distributional solutions), as follows.

Definition 8 A function $\left(u_{t}\right)_{t \geq 0} \subset \mathcal{P}(0, \infty)$ satisfies the renewal equation (2) in the distribution sense, if for all $T>0$ and all test function $\psi \in C_{\mathrm{comp}}^{1}([0, T] \times[0, \infty[)$ such that $\psi(x, T) \equiv 0$, we have

$$
-\int_{0}^{T} \int_{0}^{\infty}\left[\frac{\partial \psi(x, t)}{\partial t}+g(x) \frac{\partial \psi(x, t)}{\partial x}-d(x) \psi(x, t)+d(x) \psi(0, t)\right] u_{t}(\mathrm{~d} x) \mathrm{d} t=\int_{0}^{\infty} \psi(x, 0) u_{0}(\mathrm{~d} x) .
$$

Theorem 9 (Well posednesss) We assume (53). There is a unique weak solution of the renewal equation (2).

For the existence part, we refer to $[2,12]$ where more elaborate equations are treated. For uniqueness, need to study the inhomogeneous dual problem. We introduce a source term $S(x, t)$ on a given time interval $[0, T]$ and

$$
\left\{\begin{array}{l}
-\frac{\partial}{\partial t} \psi(x, t)-g(x) \frac{\partial}{\partial x} \psi(x, t)+d(x) \psi(x, t)=\psi(0, t) d(x)+S(x, t) \\
\psi(x, T)=0
\end{array}\right.
$$

This problem is backward in $t$ and $x$, therefore it does not use a boundary condition at $x=0$.

Lemma 10 (Existence for the dual problem) Assume (53), $S \in C_{\text {comp }}^{1}\left([0, T) \times \mathbb{R}^{+}\right)$and $d \in$ $C^{1}(0, \infty)$, then there is a unique $C^{1}$ solution to the dual equation (54). Moreover $\psi(x, t)$ vanishes for $x \geq R>0$ for some $R$ depending on the data and $T$, and the bound holds

$$
\sup _{0 \leq t \leq T, x \in \mathbb{R}^{+}}|\psi(x, t)| \leq C(T)\|S\|_{\infty} .
$$


Proof. We use the method of characteristics based on the solution of the differential system parametrized by the Cauchy data $(x, t)$ which is fixed

$$
\left\{\begin{array}{l}
\frac{d}{d s} X_{s}=g\left(X_{s}\right), \quad 0 \leq s \leq T \\
X_{t}=x \geq 0
\end{array}\right.
$$

It is well-posed thanks to the Cauchy-Lipschitz theorem and $X_{s} \geq 0$ thanks to assumption $g(0) \geq 0$. It might be useful to keep in mind that $X_{s}$ depends on $(x, t)$ and thus the notation $X_{s} \equiv X_{s}(x, t)$.

Then, we set

$$
\begin{gathered}
\widetilde{\psi}(s ; x, t)=\psi\left(s, X_{s}\right) e^{\int_{s}^{t} d\left(\sigma, X_{\sigma}\right) \mathrm{d} \sigma}, \quad \widetilde{d}(s ; x, t)=d\left(s, X_{s}\right) e^{\int_{s}^{t} d\left(\sigma, X_{\sigma}\right) \mathrm{d} \sigma}, \\
\widetilde{S}(s ; x, t)=S\left(s, X_{s}\right) e^{\int_{s}^{t} d\left(\sigma, X_{\sigma}\right) \mathrm{d} \sigma}
\end{gathered}
$$

and ignore the parameter $(x, t)$ when the statements are clear enough. We rewrite equation (54) as

$$
\begin{aligned}
\frac{d}{d s} \widetilde{\psi}(s) & =\left.\left[\frac{\partial}{\partial t} \psi+g \frac{\partial}{\partial x} \psi-d \psi\right] e^{\int_{s}^{t} d\left(\sigma, X_{\sigma}\right) \mathrm{d} \sigma}\right|_{\left(s, X_{s}\right)} \\
& =-\psi(0, s) \widetilde{d}(s)-\widetilde{S}(s)
\end{aligned}
$$

Next, we integrate between $s=t$ and $s=T$, use the Cauchy data at $t=T$ and the identity $\widetilde{\psi}(t)=\psi(x, t)$, and we obtain

$$
\psi(x, t)=\int_{t}^{T}[\psi(0, s) \widetilde{d}(s ; x, t)+\widetilde{S}(s ; x, t)] \mathrm{d} s .
$$

This integral equation can be solved first for $x=0$. Then, equation (55) is reduced to the Volterra equation

$$
\psi(0, t)=\int_{t}^{T}[\psi(0, s) \widetilde{d}(s ; 0, t)+\widetilde{S}(s ; 0, t)] \mathrm{d} s, \quad 0 \leq t \leq T
$$

which, thanks to the (backward) Cauchy-Lipschitz theorem, has a unique solution that vanishes for $t=T$. By the $C^{1}$ regularity of the data, we also have $\psi(0, t) \in C^{1}([0, T])$.

Since $\psi(0, t)$ is now known, formula (55) gives us the explicit form of the solution for all $(x, t)$. Notice that, in the compact support statement, $\widetilde{\psi}(x, t)$ vanishes for $x \geq R$ where $R$ denotes the size of the support of $S$ in $x$, plus $T\|g\|_{\infty}$. The uniform bound on $\psi$ also follows from formula (55),

Proof. [Uniqueness for the renewal equation.] With the help of the dual problem, we can use the Hilbert Uniqueness Method. The idea is simple: when the coefficients $d, g$ satisfy the assumptions of Lemma 10, we can use the solution $\psi$ of (54) as a test function in the weak formulation of Definition 8. For the difference $u=u^{2}-u^{1}$ between two possible solutions $u^{2}, u^{1}$ with the same initial data, we arrive at

$$
\int_{0}^{T} \int_{0}^{\infty}\left[\frac{\partial \psi(x, t)}{\partial t}+g(x) \frac{\partial \psi(x, t)}{\partial x}-d(x) \psi(x, t)+d(x) \psi(0, t)\right] u_{t}(\mathrm{~d} x) \mathrm{d} t=0
$$


for $\psi(\cdot, \cdot) \in C^{1}$ which is the case when $d \in C^{1}$. Then, taking into account (54), we arrive at

$$
\int_{0}^{T} \int_{0}^{\infty} S(x, t) u_{t}(\mathrm{~d} x) \mathrm{d} t=0,
$$

for all $T>0$ and all functions $S \in C_{\text {comp }}^{1}$, and this implies $u \equiv 0$.

When $d$ is merely continuous, we consider a regularized family $d_{p} \rightarrow d$ where the convergence holds locally uniformly. Then, for a given function $S \in C_{\mathrm{comp}}^{1}$, we solve (54) with $d_{p}$ in place of $d$ and call $\psi_{p}$ its solution (which is uniformly bounded with compact support). Inserting it in the definition of weak solutions, we obtain

$$
\begin{gathered}
\int_{0}^{T} \int_{0}^{\infty} S(x, t) u_{t}(\mathrm{~d} x) \mathrm{d} t=R_{p}, \\
R_{p}=\int_{0}^{T} \int_{0}^{\infty}\left[d_{p}-d(x)\right]\left[\psi_{p}(x, t)-\psi_{p}(0, t)\right] u_{t}(\mathrm{~d} x) \mathrm{d} t,
\end{gathered}
$$

and using that $\psi_{p}$ is uniformly bounded, we deduce that

$$
\left|R_{p}\right| \leq T\|\psi\|_{\infty}\left\|d_{p}-d\right\|_{\infty} \underset{p \rightarrow \infty}{\longrightarrow} 0
$$

Therefore, we have recovered the identity $\int_{0}^{T} \int_{0}^{\infty} S(x, t) u_{t}(\mathrm{~d} x) \mathrm{d} t=0$, for all functions $S \in C_{\text {comp }}^{1}$, and this implies again $u \equiv 0$.

This concludes the uniqueness result stated in Theorem 9.

\section{References}

[1] L. Ambrosio, N. Gigli, and G. Savaré, Gradient flows in metric spaces and in the space of probability measures, Lectures in Mathematics ETH Zürich, Birkhäuser Verlag, Basel, second ed., 2008 .

[2] V. Bansaye, B. Cloez, And P. Gabriel, Ergodic behavior of non-conservative semigroups via generalized Doeblin's conditions, Acta Appl. Math., 166 (2020), pp. 29-72.

[3] H. Berry, T. Lepoutre, And A. M. GonzÁlez, Quantitative convergence towards a selfsimilar profile in an age-structured renewal equation for subdiffusion, Acta Appl. Math., 145 (2016), pp. 15-45.

[4] J. Bertoin And A. R. WAtson, The strong Malthusian behavior of growth-fragmentation processes, Ann. H. Lebesgue, 3 (2020), pp. 795-823.

[5] S. Bianchini And M. Gloyer, An estimate on the flow generated by monotone operators, Comm. Partial Differential Equations, 36 (2011), pp. 777-796.

[6] F. Bouchut, F. James, And S. MAncini, Uniqueness and weak stability for multi-dimensional transport equations with one-sided Lipschitz coefficient, Ann. Sc. Norm. Super. Pisa Cl. Sci. (5), 4 (2005), pp. 1-25. 
[7] R. Bürger, The mathematical theory of selection, recombination, and mutation, Wiley Series in Mathematical and Computational Biology, John Wiley \& Sons, Ltd., Chichester, 2000.

[8] V. Calvez, P. Gabriel, and A. Mateos González, Limiting Hamilton-Jacobi equation for the large scale asymptotics of a subdiffusion jump-renewal equation, Asymptot. Anal., 115 (2019), pp. 63-94.

[9] V. Calvez, J. Garnier, and F. Patout, Asymptotic analysis of a quantitative genetics model with nonlinear integral operator, J. Éc. polytech. Math., 6 (2019), pp. 537-579.

[10] J. Chevallier, M. J. Cáceres, M. Doumic, and P. Reynaud-Bouret, Microscopic approach of a time elapsed neural model, Math. Models Methods Appl. Sci., 25 (2015), pp. 26692719 .

[11] J. M. Cushing, An introduction to structured population dynamics, vol. 71 of CBMS-NSF Regional Conference Series in Applied Mathematics, Society for Industrial and Applied Mathematics (SIAM), Philadelphia, PA, 1998.

[12] T. Debiec, M. Doumic, P. Gwiazda, and E. Wiedemann, Relative entropy method for measure solutions of the growth-fragmentation equation, SIAM J. Math. Anal., 50 (2018), pp. 58115824 .

[13] R. J. DiPerna And P.-L. Lions, Ordinary differential equations, transport theory and Sobolev spaces, Invent. Math., 98 (1989), pp. 511-547.

[14] R. L. Dobrušin, Vlasov equations, Funktsional. Anal. i Prilozhen., 13 (1979), pp. 48-58, 96.

[15] M. Doumic, M. Hoffmann, N. Krell, And L. Robert, Statistical estimation of a growthfragmentation model observed on a genealogical tree, Bernoulli, 21 (2015), pp. 1760-1799.

[16] M. Doumic, A. Olivier, And L. Robert, Estimating the division rate from indirect measurements of single cells, Discrete Contin. Dyn. Syst. Ser. B, 25 (2020), pp. 3931-3961.

[17] W. Feller, On the integral equation of renewal theory, Ann. Math. Statistics, 12 (1941), pp. 243267.

[18] L. Ferretti, C. Wymant, M. Kendall, L. Zhao, A. Nurtay, L. Abeler-Dörner, M. PARKer, D. Bonsall, AND C. Fraser, Quantifying sars-cov-2 transmission suggests epidemic control with digital contact tracing, Science, 368 (2020).

[19] N. Fournier And E. Löcherbach, On a toy model of interacting neurons, Ann. Inst. Henri Poincaré Probab. Stat., 52 (2016), pp. 1844-1876.

[20] N. Fournier and B. Perthame, Transport distances for pdes: the coupling method, EMS Surv. Math. Sci., 7 (2020), pp. 1-31.

[21] F. Golse, C. Mounot, And T. Paul, On the mean field and classical limits of quantum mechanics, Comm. Math. Phys., 343 (2016), pp. 165-205.

[22] M. Hauray, Wasserstein distances for vortices approximation of Euler-type equations, Math. Models Methods Appl. Sci., 19 (2009), pp. 1357-1384. 
[23] C. Marchioro And M. Pulvirenti, Mathematical theory of incompressible nonviscous fluids, vol. 96 of Applied Mathematical Sciences, Springer-Verlag, New York, 1994.

[24] J. A. J. Metz And O. Diekmann, The dynamics of physiologically structured populations, vol. 68 of Lecture Notes in Biomath., Springer, Berlin, 1986.

[25] S. Mirrahimi And G. Raoul, Dynamics of sexual populations structured by a space variable and a phenotypical trait, Theoretical Population Biology, 84 (2013), pp. 87-103.

[26] S. Mischler And Q. Weng, Relaxation in time elapsed neuron network models in the weak connectivity regime, Acta Appl. Math., 157 (2018), pp. 45-74.

[27] P. Monmarché, On $\mathcal{H}^{1}$ and entropic convergence for contractive PDMP, Electron. J. Probab., 20 (2015), pp. Paper No. 128, 30.

[28] S. Nordmann, B. Perthame, and C. Taing, Dynamics of concentration in a population model structured by age and a phenotypical trait, Acta Appl. Math., 155 (2018), pp. 197-225.

[29] K. Pakdaman, B. Perthame, and D. Salort, Dynamics of a structured neuron population, Nonlinearity, 23 (2010), pp. 55-75.

[30] B. Perthame, Transport equations in biology, Frontiers in Mathematics, Birkhäuser Verlag, Basel, 2007.

[31] F. Santambrogio, Optimal transport for applied mathematicians, vol. 87 of Progress in Nonlinear Differential Equations and their Applications, Birkhäuser/Springer, Cham, 2015.

[32] C. Villani, Topics in optimal transportation, vol. 58 of Graduate Studies in Mathematics, American Mathematical Society, Providence, RI, 2003. 